\title{
Semiclassical evaluation of expectation values
}

\author{
K. M. Mittal ${ }^{1,2}$, O. Giraud ${ }^{1}$, D. Ullmo ${ }^{1 *}$ \\ 1 Université Paris-Saclay, CNRS, LPTMS, 91405, Orsay, France \\ 2 Indian Institute of Science Education and Research, Dr. Homi Bhabha Road, Pune 411 \\ 008, India \\ * denis.ullmo@universite-paris-saclay.fr
}

July 28, 2020

\begin{abstract}
Semiclassical mechanics allows for a description of quantum systems which preserves their phase information, and thus interference effects, while using only the system's classical dynamics as an input. In particular one of the strengths of a semiclassical description is to present a coherent picture which (up to negligible higher-order $\hbar$ corrections) is independent of the particular canonical coordinates used. This coherence relies however heavily on the use of the stationary phase approximation, which appears in many circumstances as the building block of the semiclassical approach.

It turns out however that in some (important) cases, a brutal application of stationary phase approximation washes out all interference (and thus quantum) effects. This rather basic, but in the end quite subtle, point has actually generated quite a bit of confusion in the semiclassical literature. In this paper, we address this issue in detail in one of its simplest instantiations, namely the evaluation of the time evolution of the expectation value of an operator. We explain why it is necessary to include contributions which are not in the neighborhood of stationary points and provide new semiclassical expressions for the evolution of the expectation values. Along the way, we get a deeper understanding of the origin of these interference effects and an intuitive geometric picture associated with them.
\end{abstract}




\section{INTRODUCTION}

Semiclassical approximations form a family of techniques, including for instance the van Vleck approximation of the quantum evolution operator [1], the time-dependent WKB approximation for the propagation of wave functions [2], or the Gutzwiller trace formula for the density of states [3, 4], making it possible to describe quantitatively a quantum system in a certain range of parameters, using only an input from the corresponding classical dynamics. In our nomenclature classical approximations of a quantum quantity are approximations in which interference effects are ignored, or lost. By contrast, semiclassical approximations are such that information about phases is kept, and thus provide a description of interference effects.

The stationary phase approximation plays a central role in semiclassical approaches. Indeed, common tools in quantum mechanics, such as the Fourier transform or the Wigner transform, involve integrals of the form

$$
\int d^{K} \mathbf{q} A(\mathbf{q}) e^{\frac{i}{\hbar} S(\mathbf{q})}
$$

where the integrand has a smooth envelope $A(\mathbf{q})$ and a rapidly oscillating phase $S(\mathbf{q}) / \hbar$. For relatively deep reasons, semiclassical approximations usually keep the leading-order term and the first-order correction in $\hbar$, but neglect higher-order corrections, which is exactly what the stationary phase approximation does. Within the semiclassical framework, integrals such as (1) are thus essentially always performed within the stationary phase approximation, and the understanding that any such integral has to be performed within this approximation actually provides a global coherence to the semiclassical pictures. Consider for example the van Vleck formula expressing the Feynman propagator as a sum over classical trajectories. It can be derived through a WKB approximation "à la Maslov" [5], where one looks for solutions of partial differential equations in the form of formal series in $\hbar$. It can also be obtained from the path integral representation of the exact quantum evolution operator if all integrals in the path integral formalism are performed within the stationary phase approximation [6]. Both approaches lead to exactly the same result.

One of the great strengths of semiclassical approaches is the possibility to freely choose the system of coordinates suited to our needs. Indeed, one can show that semiclassical approximations keep the same form under a canonical change of the classical variables within the stationary phase approximation. This property is used in particular by Maslov [5] to 
treat cases where the amplitude $A(\mathbf{q})$ in (1) may become singular, as we shall see later in this paper.

Because of the identity between expressions obtained from the Maslov $\hbar$ expansion and the stationary phase approximation, there is, in the mind of many practitioners of the field, a kind of identification between semiclassical and stationary phase approximations. There are of course some limitations to this identification, as in some circumstances, e.g. near turning points or caustics, or near the bifurcation of a periodic orbit, a naive application of the stationary phase approximation leads to divergences. These have to be cured by uniform approximations, in which the behavior of the neighborhood of stationary phase point is described more carefully than the usual quadratic approximation. It remains nevertheless that in almost all circumstances, only the neighborhood of the stationary phase points can contribute significantly to an integral of the form (1), and within a semiclassical approach to a physical problem this is most of the time implicitly or explicitly assumed.

There is however a class of problems for which this rather natural assumption turns out to fail. This class includes relatively "involved" physical quantities, like fidelity [7-9], OTOC [10, 11], or the quantum corrections to the conductance in mesocopic quantum dots [12, 13], but also some others as benign as the time evolution of the expectation value $\langle\hat{O}\rangle=\langle\Psi|\hat{O}| \Psi\rangle$ of an operator $\hat{O}$ for some time-dependent quantum state $\left|\Psi_{t}\right\rangle$ [14], or more general correlation functions [15]. In this paper, we shall focus on the simple case of the expectation value.

To be more specific, consider a $K$-dimensional dynamical system, whose phase-space $\mathcal{M}$ is the set of points $\mathbf{x}=(\mathbf{p}, \mathbf{q})$ with $\mathbf{p}=\left(p_{1}, \ldots, p_{K}\right)$ and $\mathbf{q}=\left(q_{1}, \ldots, q_{K}\right)$. Suppose the system is initially described by a state $\left|\Psi_{0}\right\rangle$, which evolves under some Hamiltonian dynamics to a state $\left|\Psi_{t}\right\rangle$ at time $t$. The Wigner transform $O(\mathbf{x})$ of an arbitrary operator $\hat{O}$ is defined by

$$
O(\mathbf{x})=\frac{1}{(2 \pi \hbar)^{K}} \int d^{K} \mathbf{q}^{\prime} e^{-\frac{i}{\hbar} \mathbf{p} \cdot \mathbf{q}^{\prime}}\left\langle\mathbf{q}+\frac{\mathbf{q}^{\prime}}{2}|\hat{O}| \mathbf{q}-\frac{\mathbf{q}^{\prime}}{2}\right\rangle, \quad \mathbf{x} \in \mathcal{M}
$$

We denote by $W_{t}(\mathbf{x})$ the Wigner transform of the state $\left|\Psi_{t}\right\rangle$, namely

$$
W_{t}(\mathbf{x})=\frac{1}{(2 \pi \hbar)^{K}} \int d^{K} \mathbf{q}^{\prime} e^{-\frac{i}{\hbar} \mathbf{p} \cdot \mathbf{q}^{\prime}} \overline{\Psi_{t}}\left(\mathbf{q}-\frac{\mathbf{q}^{\prime}}{2}\right) \Psi_{t}\left(\mathbf{q}+\frac{\mathbf{q}^{\prime}}{2}\right),
$$

where bar denotes complex conjugation. The expectation value $\langle\hat{O}\rangle(t)=\left\langle\Psi_{t}|\hat{O}| \Psi_{t}\right\rangle$ can then be written [16] as the phase-space integral

$$
\langle\hat{O}\rangle(t)=\int d^{K} \mathbf{x} W_{t}(\mathbf{x}) O(\mathbf{x})
$$


Suppose $\hat{O}$ is "smooth", in the sense that its Wigner transform varies only on the classical scale. Then one can show that keeping only the contribution of the neighborhood of stationary phase points in the integral of Eq. (4) leads to [17]

$$
\langle\hat{O}\rangle_{\mathrm{cl}}(t)=\int d^{K} \mathbf{x} W_{0}(\mathbf{x}) O\left(g_{t} \mathbf{x}\right)=\int d^{K} \mathbf{x} W_{0}\left(g_{t}^{-1} \mathbf{x}\right) O(\mathbf{x}),
$$

with $g_{t}$ the classical Hamiltonian flow characterizing the classical dynamics associated with the Hamiltonian $\hat{H}$. In words, $\langle\hat{O}\rangle_{\mathrm{cl}}(t)$ is obtained as the overlap of the Wigner transform of the operator $\hat{O}$ with the classically evolved Wigner transform of the state $|\Psi\rangle$. This expression is often referred to as the Linearized Semiclassical Initial Value Representation (LSC-IVR) [18] in chemical or molecular physics, or the Truncated Wigner Approximation (TWA) in the context of cold atoms [19], but can be tracked back at least to the work of Heller [20] in 1976 and is discussed in the review written by Wigner and co-authors [21] in 1984. Within this approximation, all interference effects are washed out, and the expression (5) yields essentially the classical approximation to the mean value.

This result actually leads us to consider several possibilities. The first one would be that either interference effects are indeed completely washed out, and that for the expectation value of any smooth operator, the classical approximation Eq. (5) is indeed a correct description of the quantum evolution; or that rapidly oscillating corrections to this classical terms do exist but that they are beyond the reach of semiclassics. There is however a large literature, starting from the seminal paper of Tomsovic and Heller [22], and including some situations for which the issue of non-applicability of the stationary phase approximation exist [7-13, 23, 24], which shows that on a quite general basis the interference effects that set on after the Ehrenfest time can be described within a semiclassical approach, and that their effects is non negligible. We therefore clearly also expect this to be the case for the mean value of smooth operators [14, 15].

All the examples above provide a clear demonstration of the effectiveness of semiclassics. However, the physical problem they describe, and the approach they use, often involve either a statistical argument [7-13, 23] or, in the spirit of the Initial Value Representation (IVR), to perform numerically the integral for which the stationary phase approximation fails [24]. Therefore, the rationale dictating when stationary phase can be used and when it cannot is not always absolutely clear, and one may be worried about the fact that this necessity to do without stationary phase approximation in some circumstances but not in others 
could harm the general coherence of the semiclassical approach. The goal of our work is to clarify these issues on the simple case of the operator mean value. Our aim is to provide a semiclassical description of interference effects for the time evolution (4) of the mean value of a smooth operator $\hat{O}$. We shall assume that $\hat{O}$ is smooth in the sense that its Wigner transform varies only on classical scales, that is, shows no short-scale quantum features. In particular we will clarify why in the integral of Eq. (4) one should keep contributions which are not in the neighborhood of a stationary phase point. Along the way, we shall gain a deeper understanding of the origin of the interference terms in the time-dependent expectation values of operators, as well as a new semiclassical expression for them which, for some (simple enough) operators $\hat{O}$, are rather explicit.

The structure of the paper is as follows. We start, in the two following sections, by reviewing two important tools of semiclassical theory that we will need for our discussion. We begin in section II by a presentation of the Maslov approach [5] which relates the semiclassical evolution of a quantum wavefunction to the classical evolution of the Lagrangien manifold on which it is constructed. We then derive in section III an expression for the semiclassical Wigner function, which is the starting point of our discussion of the expectation value of smooth operators. This derivation will follow very closely the spirit of the original work of Berry [25]. However, we shall express this semiclassical Wigner function in a slightly different form, which transforms under canonical change of variable in a rather transparent way. This will in particular make it possible to show that the relevant interference terms in the computation of a smooth operator's mean value are expressed only in terms of canonical invariants. Relying on this canonical invariance of the formalism, we then address in section IV the question of the interference terms in the expectation value of a smooth operator, discuss their origin, the precise place where stationary phase approximation cannot be used (and why), and provide an expression for these interference terms which turns out to be in the end rather intuitive and natural. Finally, we conclude in section $\square$ with a comparison between the point of view taken in this paper and the approach suggested by an Initial Value Representation (IVR) approach. 


\section{SEMICLASSICAL WAVEFUNCTIONS}

\section{A. Wavefunctions from Lagrangian manifolds}

We assume in this paper that the initial state we consider is a semiclassical wavefunction, that is, a state of the form $\psi_{0}(\mathbf{q})=\mathcal{A}(\mathbf{q}) \exp (i S(\mathbf{q}) / \hbar)$, or possibly a finite sum of terms of that form. Such a function is the product of a smooth envelope $\mathcal{A}(\mathbf{q})$ and a function that oscillates rapidly when $\hbar \ll 1$. Provided one extend this notion to include complex Lagrangian manifolds so that coherent states can be treated within this framework [26], all the quantum states of interest in semiclassical physics in practice fall in that category.

A procedure introduced by Maslov [5], that we briefly sketch now, allows to associate such a function with a Lagrangian manifold in the classical phase space. We stress that we do not introduce a specific dynamics/Hamiltonian yet, and the semiclassical wavefunction that we are going to build from the Lagrangian manifold is a priori not the eigenstate of a specific model, but should be viewed as the possible initial state of some quantum evolution to be specified later. The Lagrangian manifolds that we consider here are $K$-dimensional manifolds in the $2 K$-dimensional phase space $\mathcal{M}$, that are characterized by the existence of a function $S(\mathbf{q})$ such that the $\mathbf{p}$ coordinates can be expressed as the gradient of that function, namely $\mathbf{p}=\partial S / \partial \mathbf{q}$ (Theorem 4.20 of [5]).

A natural way to construct a Lagrangian manifold from state $\psi_{0}(\mathbf{q})=\mathcal{A}(\mathbf{q}) \exp (i S(\mathbf{q}) / \hbar)$ is to consider the set $\mathcal{L}$ of phase-space points such that $\mathbf{p}=\partial S / \partial \mathbf{q}$ : by definition it is a Lagrangian manifold. Conversely, for any Lagrangian manifold $\mathcal{L}$, and given any smooth function $\mathcal{A}(\mathbf{q})$, one can construct the semiclassical state $\mathcal{A}(\mathbf{q}) \exp \left(i S_{\mathcal{L}}(\mathbf{q}) / \hbar\right)$ with $S_{\mathcal{L}}(\mathbf{q}) \equiv$

$\int_{\gamma: \mathbf{x}_{0} \rightarrow \mathbf{x}} \mathbf{p} d \mathbf{q}$, where $\gamma$ is any path on $\mathcal{L}$ going from an arbitrary $\mathbf{x}_{0}$ to $\mathbf{x}=(\mathbf{p}, \mathbf{q})$. Indeed, a consequence of the fact that $\mathcal{L}$ is Lagrangian is that the integral defining $S_{\mathcal{L}}(\mathbf{q})$ does not depend on the path of integration but only on the endpoints.

\section{B. One-dimensional case}

As an illustration, let us consider the one-dimensional case $K=1$. The phase space $\mathcal{M}$ is now two-dimensional, and a one-dimensional Lagrangian manifold $\mathcal{L}$ is simply a curve in phase space, specified by some function $S_{\mathcal{L}}(q)$ as the set of points $(p, q)$ with $p=d S_{\mathcal{L}}(q) / d q$. A path $\gamma$ on $\mathcal{L}$ is a portion of that curve connecting an initial point $x_{0}$ to a final point $x$, 
and the function $S_{\mathcal{L}}(q)$ can be expressed as $S_{\mathcal{L}}(q)=\int_{\gamma: x_{0} \rightarrow x} p d q$, with $x_{0}$ arbitrarily fixed on $\mathcal{L}$. We parameterize the curve $\mathcal{L}$ as $\mathcal{L}=\{x(s), s \in[0,1]\}$, with of course $x(s)=(p(s), q(s))$, and possibly $x(0)=x(1)$. We then associate with $\mathcal{L}$ a semiclassical wavefunction following the procedure indicated above (see Fig. 1 for an illustration). Choosing a prefactor of the form $\mathcal{A}(q)=a(s) / \sqrt{|d q / d s|}$ in a domain where $q(s)$ is monotonous (thus invertible), we set

$$
\psi_{\mathcal{L}}(q)=\frac{a(s)}{\sqrt{|d q / d s|}} \exp \left(\frac{i}{\hbar} S_{\mathcal{L}}(q)-i \frac{\pi}{2} \mu\right), \quad S_{\mathcal{L}}(q)=\int_{\gamma: x_{0} \rightarrow x} p(s) \frac{d q}{d s} d s,
$$

where $a:[0,1] \rightarrow \mathbb{C}$ is some smooth function of $s$, evaluated at the parameter value corresponding to $q$, and the index $\mu$, introduced for later convenience, is a Maslov index which is zero in this simple case. To go from $q$ to $p$ representation we define the Fourier transform of a function $\psi(q)$ as

$$
\tilde{\psi}(p)=\frac{1}{(2 i \pi \hbar)^{1 / 2}} \int d q e^{-\frac{i}{\hbar} p q} \psi(q)
$$

The Fourier transform (7) has the property that $|\tilde{\psi}(p)|^{2}|\partial p / \partial q|=|\psi(q)|^{2}|\partial q / \partial p|$ up to $1 / \hbar$ corrections. If we calculate the Fourier transform $\tilde{\psi}_{\mathcal{L}}(p)$ of $\psi_{\mathcal{L}}(q)$ in the stationary phase approximation, we get that the stationary point is at a value of $q$ such that $d S_{\mathcal{L}} / d q=p$, and Eq. (7) then gives

$$
\tilde{\psi}_{\mathcal{L}}(p)=\frac{a(s)}{\sqrt{|d p / d s|}} \exp \left(\frac{i}{\hbar} \tilde{S}_{\mathcal{L}}(p)-i \frac{\pi}{2} \tilde{\mu}\right), \quad \tilde{S}_{\mathcal{L}}(p)=-\int_{\mathcal{L}: x_{0} \rightarrow x} q(s) \frac{d p}{d s} d s
$$

where $\tilde{\mu}=1$ if $d p / d q=d^{2} S_{\mathcal{L}} / d q^{2}$ is negative and 0 otherwise (Corollary 1.9 of [5]). Here the prefactor $a(s)$ is evaluated at the parameter value $s$ corresponding to the stationary point $q$ at which $d S_{\mathcal{L}} / d q=p$, that is, at the parameter value $s$ corresponding to $p$ itself. Remarkably, because of the choice of the form of the prefactor $\mathcal{A}(q)$, the semiclassical wavefunctions (6) and (8) have exactly the same form, up to the sign in front of the action.

Expression (8) is valid only in the case where $d p / d s \neq 0$. Of course, there is no special role played by variable $q$ in the above construction, and one can as well directly associate with $\mathcal{L}$ a semiclassical wavefunction in the $p$ representation. Such a function can be obtained by changing $(p, q)$ to $(q,-p)$ in Eq. (6), and it will be defined in a domain where $p(s)$ is invertible. In fact, this function exactly takes the form (8), and therefore we denote it by $\tilde{\psi}_{\mathcal{L}}(p)$. Thus, the association between a manifold $\mathcal{L}$ and a wavefunction $\psi_{\mathcal{L}}$ has this nice property that it can be performed in a symmetric way in the $q$ or the $p$ representation.

If both $q(s)$ and $p(s)$ are invertible, it is equivalent (up to a constant phase factor) to obtain the semiclassical wavefunction using either representation. For a generic $\mathcal{L}$ however, 


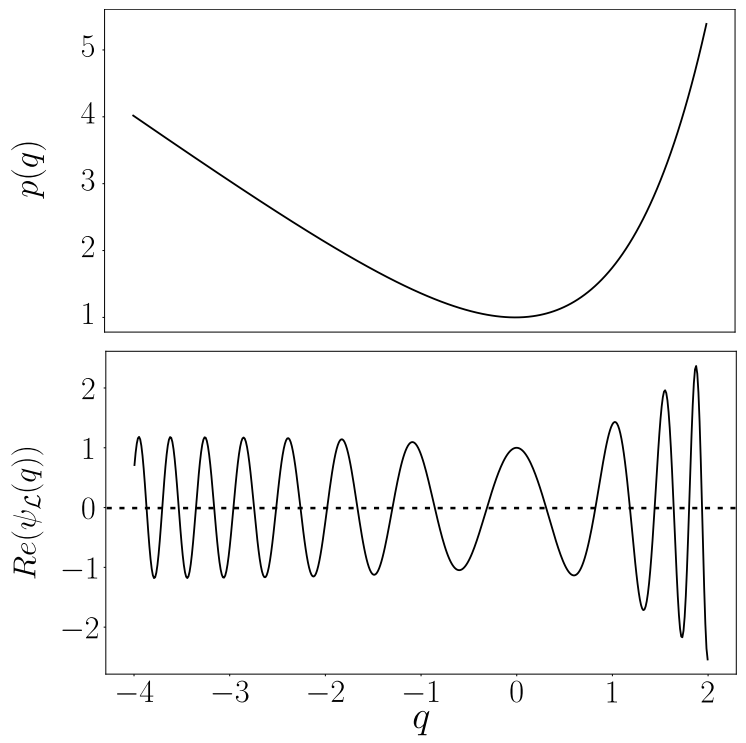

FIG. 1. Example of the association of a semiclassical wavefunction with a Lagrangian manifold. Top: the "Lagrangian manifold" (here, for $K=1$ just a curve) defined by $p(q)=e^{q}-q$. Bottom: real part of the associated wavefunction, assuming $a(s)=1$ with the parameter $s$ chosen as the curvilinear distance along the curve (i.e. $d s^{2}=d q^{2}+d p^{2}$ ) and a value of $\hbar=0.2$.

neither $q(s)$ nor $p(s)$ will be invertible over the whole parameter range (think of an ellipse parameterized by an angle). The procedure suggested by Maslov is then to introduce a partition of the unity, i.e. a set of $n$ smooth positive real functions $\left\{\varphi_{\alpha}(s), \alpha=1, \ldots, n\right\}$ such that $\sum_{1}^{n} \varphi_{\alpha}(s)=1$, and to choose it such that for any $\alpha$, either $q(s)$ or $p(s)$ is invertible on the support of $\varphi_{\alpha}$. In that case one can define a function $\psi_{\alpha}(q)$ in $q$ representation using either Eq. (6) (if $q(s)$ is invertible) or the inverse Fourier transform of Eq. (8) (if $p(s)$ is invertible), in both cases with amplitude $\varphi_{\alpha}(s) a(s)$. The semiclassical wavefunction associated with $\mathcal{L}$ is then simply $\psi_{\mathcal{L}}(q)=\sum_{\alpha} \psi_{\alpha}(q)$. It can then be shown that if in Eqs. (6)(8) the indices $\mu_{\alpha}, \tilde{\mu}_{\alpha}$ count the (algebraic) number of caustics from $x_{0}$ to the support of $\varphi_{\alpha}$ for the corresponding representation, the resulting wavefunction is, up to negligible higher order in $\hbar$ corrections, independent on the details of the choice of the $\varphi_{\alpha}$, and in particular that the phase is a continuous function.

If $\mathcal{L}$ is not a closed curve, this completes the construction. If $x(0)=x(1)$ however, one needs to further impose that the total phase is single-valued, which leads to the usual EBK 
quantization condition

$$
\oint_{\mathcal{L}} p d q=\left(n+\mu_{\mathcal{L}} / 4\right) 2 \pi \hbar
$$

with $\mu_{\mathcal{L}}$ the Maslov index associated with $\mathcal{L}$ (which is usually 2 when $K=1$ ). We stress again that we did not introduce any dynamics yet (i.e. we did not specify the Hamiltonian, which could for instance be a time-dependent one), and the EBK condition (9) does not specify an eigenstate of the problem, but just a constraint on the manifold $\mathcal{L}$ such that one can associate to it a wavefunction using the Maslov procedure.

\section{Generalization to arbitrary dimension}

This construction generalizes straightforwardly to the general case of a $K$-dimensional system. The symplectic structure of phase space $\mathcal{M}$ can be introduced through the wedge product giving the symplectic area of a pair of phase-space vectors $\left(\mathbf{x}, \mathbf{x}^{\prime}\right)$,

$$
\mathbf{x} \wedge \mathbf{x}^{\prime}=\sum_{i=1}^{K}\left(p_{i} q_{i}^{\prime}-q_{i} p_{i}^{\prime}\right)=\left(\mathbf{x}^{\prime}\right)^{T} J \mathbf{x}, \quad J=\left(\begin{array}{cc}
0 & -\mathbb{1} \\
\mathbb{1} & 0
\end{array}\right)
$$

where $\mathbb{1}$ is the $K \times K$ identity matrix. A Lagrangian manifold is then a manifold on which the differential 2-form $\omega^{2}=\sum_{i=1}^{K} d p_{i} \wedge d q_{i}$ is uniformly zero. As a consequence, the

integral of the 1 -form $\mathbf{p} d \mathbf{q} \equiv \sum_{i=1}^{K} p_{i} d q_{i}$ along any path on $\mathcal{L}$ is invariant under a continuous deformation of that path with fixed endpoints.

If $\mathcal{L}$ is $K$-dimensional, we can parameterize it by a vector of parameters $\mathbf{s} \in \mathbb{R}^{K}$. Formulas (6)-(9) are easily adapted if we use the compact notation where $\partial \mathbf{q} / \partial$ s denotes the $K \times K$ matrix with matrix elements $\partial q_{i} / \partial s_{j}$ and $\partial S / \partial \mathbf{q}$ denotes the vector with components $\partial S / \partial q_{i}$. Then the Lagrangian manifold $\mathcal{L}$ is specified by some function $S_{\mathcal{L}}(\mathbf{q})$, and (6) becomes

$$
\psi_{\mathcal{L}}(\mathbf{q})=\frac{a(\mathbf{s})}{\sqrt{|\partial \mathbf{q} / \partial \mathbf{s}|}} \exp \left(\frac{i}{\hbar} S_{\mathcal{L}}(\mathbf{q})-i \frac{\pi}{2} \mu\right), \quad S_{\mathcal{L}}(\mathbf{q})=\int_{\gamma: \mathbf{x}_{0} \rightarrow \mathbf{x}} \mathbf{p}(\mathbf{s}) \frac{\partial \mathbf{q}}{\partial \mathbf{s}} d \mathbf{s}
$$

where |.| denotes the absolute value of the determinant of the matrix. Since $\mathcal{L}$ is Lagrangian, the action integral in (11) is invariant under a continuous deformation of the path of integration on $\mathcal{L}$ and thus, once EBK conditions such as $(9)$ are fulfilled for all generators of the homotopy group of $\mathcal{L}$, phase factors only depend on the point $\mathbf{x} \in \mathcal{L}$ but not on the path chosen to define the action integral.

At each point a $K$-dimensional Lagrangian manifold $\mathcal{L}$ is locally diffeomorphic to a coordinate $K$-dimensional plane (the $\mathbf{q}$ plane, the $\mathbf{p}$ plane, or a mixture of both) (Proposition 
4.6 of [5]), which allows to always choose local coordinates such that the parametrization is non-singular, so that one can always construct either the semiclassical wavefunction (6), or (8), or obtain an analog expression in a mixed representation.

\section{SEMICLASSICAL WIGNER FUNCTION}

In this section, we derive a semiclassical expression for the Wigner function, which will be the starting point of our discussion in section IV] We follow here very closely the spirit of the original work of Berry [25], although the precise expressions we shall obtain will take a slightly different form, whose transformation under canonical changes of variables will be somewhat more transparent.

\section{A. Stationary phase approximation}

The Wigner transform $W_{\mathcal{L}}(\mathbf{x})$ of a semiclassical wavefunction $\psi_{\mathcal{L}}$ of the form (11) is obtained using the definition (3). In order to obtain the semiclassical expression for $W_{\mathcal{L}}(\mathbf{x})$, we need to evaluate the integral

$$
W_{\mathcal{L}}(\mathbf{x})=\frac{1}{(2 \pi \hbar)^{K}} \int d^{K} \mathbf{q}^{\prime} e^{-\frac{i}{\hbar} \mathbf{p} \cdot \mathbf{q}^{\prime}} \overline{\mathcal{A}\left(\mathbf{q}-\frac{\mathbf{q}^{\prime}}{2}\right)} \mathcal{A}\left(\mathbf{q}+\frac{\mathbf{q}^{\prime}}{2}\right) e^{\frac{i}{\hbar} S_{\mathcal{L}}\left(\mathbf{q}+\frac{\mathbf{q}^{\prime}}{2}\right)-\frac{i}{\hbar} S_{\mathcal{L}}\left(\mathbf{q}-\frac{\mathbf{q}^{\prime}}{2}\right)-i \frac{\pi}{2}\left(\mu_{\mathbf{q}+\mathbf{q}^{\prime} / 2}-\mu_{\mathbf{q}-\mathbf{q}^{\prime} / 2}\right)}
$$

in the stationary phase approximation [25].

At a given $\mathbf{x}=(\mathbf{p}, \mathbf{q})$, a stationary point in the integral of Eq. 12 is reached when $\mathbf{q}^{\prime}$ takes a value $\tilde{\mathbf{q}}$ such that $\mathbf{p}=\frac{1}{2}\left[\partial S_{\mathcal{L}} /\left.\partial \mathbf{q}\right|_{\mathbf{q}-\tilde{\mathbf{q}} / 2}+\partial S_{\mathcal{L}} /\left.\partial \mathbf{q}\right|_{\mathbf{q}+\tilde{\mathbf{q}} / 2}\right]$. Symmetrically, a stationary point is also reached at $\mathbf{q}^{\prime}=-\tilde{\mathbf{q}}$. Let $\mathbf{q}^{+} \equiv \mathbf{q}+\tilde{\mathbf{q}} / 2$. According to the above definitions, $\mathbf{p}^{+}=\left(\partial S_{\mathcal{L}} / \partial \mathbf{q}\right)_{\mid \mathbf{q}^{+}}$defines a point $\mathbf{x}^{+}=\left(\mathbf{p}^{+}, \mathbf{q}^{+}\right)$on $\mathcal{L}$, corresponding to a parameter value $\mathbf{s}_{+}$. Similarly, if $\mathbf{q}^{-} \equiv \mathbf{q}-\tilde{\mathbf{q}} / 2$, then $\mathbf{p}^{-}=\left(\partial S_{\mathcal{L}} / \partial \mathbf{q}\right)_{\mid \mathbf{q}^{-}}$defines a point $\mathbf{x}^{-}=\left(\mathbf{p}^{-}, \mathbf{q}^{-}\right)$on $\mathcal{L}$, corresponding to a parameter value $\mathbf{s}_{-}$. The stationary condition can then be rewritten as $\mathbf{p}=\frac{1}{2}\left(\mathbf{p}^{+}+\mathbf{p}^{-}\right)$. Since obviously $\frac{1}{2}\left(\mathbf{q}^{+}+\mathbf{q}^{-}\right)=\mathbf{q}$, we have that $\mathbf{x}=\frac{1}{2}\left(\mathbf{x}^{+}+\mathbf{x}^{-}\right)$. Therefore, for a stationary point to exist in the integral evaluating $W_{\mathcal{L}}(\mathbf{x})$, the point $\mathbf{x}$ has to be the center of a pair of points $\left(\mathbf{x}^{+}, \mathbf{x}^{-}\right)$lying on $\mathcal{L}$. We shall below refer to $\boldsymbol{\xi}=\left(\mathbf{p}^{+}-\mathbf{p}^{-}, \mathbf{q}^{+}-\mathbf{q}^{-}\right)$ as the corresponding chord.

We find it convenient to denote with superscripts \pm all quantities evaluated at point $\mathbf{q}^{+}$ or $\mathbf{q}^{-}$on $\mathcal{L}$, parameterized by the parameter value $\mathbf{s}^{ \pm}$. All quantities at the stationary point 
can then be expressed as functions of $\mathbf{q}^{+}$and $\mathbf{q}^{-}$, or equivalently of $\mathbf{s}^{+}$and $\mathbf{s}^{-}$. At the stationary point $\mathbf{q}^{\prime}=\tilde{\mathbf{q}}$, the phase of the integrand in Eq. 12 is $\mathcal{S}(\mathbf{x}) / \hbar$ with

$$
\mathcal{S}(\mathbf{x})=S_{\mathcal{L}}\left(\mathbf{q}^{+}\right)-S_{\mathcal{L}}\left(\mathbf{q}^{-}\right)-\mathbf{p} \tilde{\mathbf{q}}=\oint_{\mathbf{x}^{-}}^{\mathbf{x}^{+}} \mathbf{p}(\mathbf{s}) \frac{\partial \mathbf{q}}{\partial \mathbf{s}} d \mathbf{s}
$$

where in the right-hand side the integral is taken along the closed contour made of any path going from $\mathbf{x}^{-}$to $\mathbf{x}^{+}$on $\mathcal{L}$ and the straight line from $\mathbf{x}^{+}$back to $\mathbf{x}^{-}$(see Fig. 2). The Hessian matrix $\partial^{2} S_{\mathcal{L}} / \partial \mathbf{q}^{2}=\partial \mathbf{p} / \partial \mathbf{q}$, evaluated at $\mathbf{q}^{+}$, is denoted by $\partial \mathbf{p}^{+} / \partial \mathbf{q}^{+}$. We define the matrix

$$
\left.R \equiv \frac{\partial^{2} S_{\mathcal{L}}}{\partial \mathbf{q}^{2}}\right|_{\mathbf{q}^{+}}-\left.\frac{\partial^{2} S_{\mathcal{L}}}{\partial \mathbf{q}^{2}}\right|_{\mathbf{q}^{-}}=\left(\frac{\partial \mathbf{p}^{+}}{\partial \mathbf{q}^{+}}\right)^{T}-\left(\frac{\partial \mathbf{p}^{-}}{\partial \mathbf{q}^{-}}\right)
$$

(since the Hessian is a symmetric matrix, we may or may not take the transpose in the right-hand side of (14)). Let $\eta$ denote the number of negative eigenvalues of $R$. At the stationary point, Gaussian integration yields

$$
W_{\mathcal{L}}(\mathbf{x})=\left(\frac{2}{\pi \hbar}\right)^{K / 2} \sum_{\beta} \frac{\mathcal{A}\left(\mathbf{q}_{\beta}^{+}\right) \mathcal{A}\left(\mathbf{q}_{\beta}^{-}\right)}{\sqrt{\left|R_{\beta}\right|}} \exp \left(\frac{i}{\hbar} \mathcal{S}_{\beta}(\mathbf{x})-i \frac{\pi}{2} \mu_{\beta}+i \eta_{\beta} \frac{\pi}{4}\right)+\text { c.c. }
$$

where the complex conjugate c.c. corresponds to the contribution of $\mathbf{q}^{\prime}=-\tilde{\mathbf{q}}[25]$. In (15) the sum runs over all stationary phase points $\pm(\tilde{\mathbf{q}})_{\beta}$, that is, all chords $\boldsymbol{\xi}_{\beta}$ such that the two points $\mathbf{x}_{\beta}^{-}=\mathbf{x}-\boldsymbol{\xi}_{\beta} / 2$ and $\mathbf{x}_{\beta}^{+}=\mathbf{x}+\boldsymbol{\xi}_{\beta} / 2$ associated with the center $\mathbf{x}$ and the chord $\boldsymbol{\xi}_{\beta}$ lie

on $\mathcal{L}$. In Eq. (19), $\mu_{\beta}=\mu_{\beta}^{+}-\mu_{\beta}^{-}$is the Maslov index computed along the path on $\mathcal{L}$ from $\mathbf{x}_{\beta}^{-}$ to $\mathbf{x}_{\beta}^{+}$. The phase $\mathcal{S}_{\beta}(\mathbf{x})$ can be interpreted as the symplectic area enclosed by the path from $\mathbf{x}_{\beta}^{-}$to $\mathbf{x}_{\beta}^{+}$and the chord $\boldsymbol{\xi}_{\beta}$. As a consequence, its variation $\delta \mathcal{S}_{\beta}$ when the phase point $\mathbf{x}$ is moved by $\delta \mathbf{x}$ is, at first order in this small quantity, the symplectic area of the quadrilateral formed by the chord $\boldsymbol{\xi}_{\beta}$ and the displacement $\delta \mathbf{x}$. We thus have $\delta \mathcal{S}_{\beta}=\boldsymbol{\xi}_{\beta} \wedge \delta \mathbf{x}=(\delta \mathbf{x})^{T} . J \boldsymbol{\xi}_{\beta}$, and therefore

$$
\frac{\partial \mathcal{S}_{\beta}}{\partial \mathbf{x}}=J \boldsymbol{\xi}_{\beta}
$$

\section{B. Canonical invariance of the Wigner function}

The "exact" quantum-mechanical Wigner function is invariant under symplectic maps, that is linear canonical transformations [27, 28]. However this is not entirely visible from the definition Eq. (3), and is often not very transparent either for semiclassical approximations of the Wigner function (see however [28], in which the amplitude of the semiclassical Wigner 


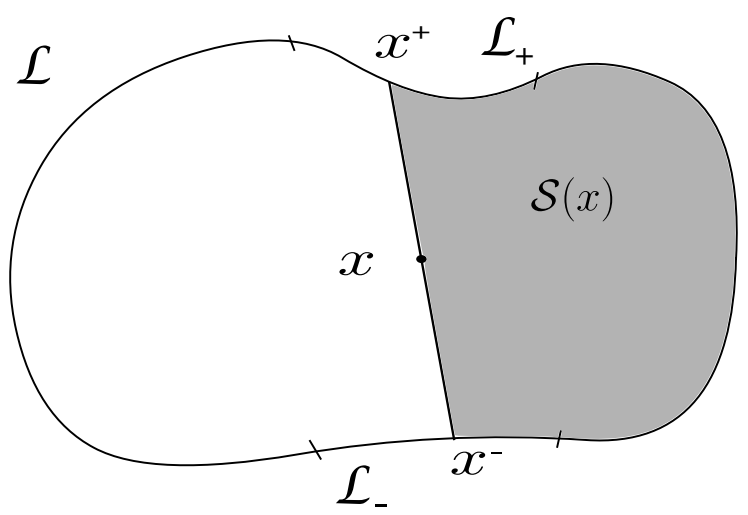

FIG. 2. Construction of the action $\mathcal{S}(\mathbf{x})$ introduced in Eq. $(13)$. For a given $\mathbf{x}$, one needs to locate two points $\mathbf{x}^{+}$and $\mathbf{x}^{-}$on the manifold $\mathcal{L}$ such that $\mathbf{x}$ is the center of the segment $\left[\mathbf{x}^{-}, \mathbf{x}^{+}\right]$ (more than one pair of points may fulfill that condition; such pairs are indexed by $\beta$ in the text). The action $\mathcal{S}(\mathbf{x})$ then corresponds to the shaded area (note that because of the EBK quantization condition Eq. (9), which side of the chord is used is irrelevant). $\mathcal{L}_{+}$and $\mathcal{L}_{-}$are pieces of $\mathcal{L}$ on which the endpoints $\mathbf{x}^{+}$and $\mathbf{x}^{-}$lie.

function is expressed in terms of Poisson brackets of a pair of actions). In this subsection we will cast (15) in a form which is manifestly invariant under linear canonical transformations.

Indeed, using the fact that $\mathcal{A}(\mathbf{q})=a(\mathbf{s}) / \sqrt{|\partial \mathbf{q} / \partial \mathbf{s}|}$, the denominator in 15 can be written in terms of the $K \times K$ matrix $D$ defined as

$$
D \equiv\left(\frac{\partial \mathbf{q}^{+}}{\partial \mathbf{s}^{+}}\right)^{T}\left[\left(\frac{\partial \mathbf{p}^{+}}{\partial \mathbf{q}^{+}}\right)^{T}-\left(\frac{\partial \mathbf{p}^{-}}{\partial \mathbf{q}^{-}}\right)\right]\left(\frac{\partial \mathbf{q}^{-}}{\partial \mathbf{s}^{-}}\right)=\left(\frac{\partial \mathbf{p}^{+}}{\partial \mathbf{s}^{+}}\right)^{T}\left(\frac{\partial \mathbf{q}^{-}}{\partial \mathbf{s}^{-}}\right)-\left(\frac{\partial \mathbf{q}^{+}}{\partial \mathbf{s}^{+}}\right)^{T}\left(\frac{\partial \mathbf{p}^{-}}{\partial \mathbf{s}^{-}}\right)
$$

and which components are given by

$$
D_{a b}=\frac{\partial \mathbf{x}^{+}}{\partial s_{a}^{+}} \wedge \frac{\partial \mathbf{x}^{-}}{\partial s_{b}^{-}} .
$$

The semiclassical Wigner function can then be rewritten as

$$
W_{\mathcal{L}}(\mathbf{x})=\left(\frac{2}{\pi \hbar}\right)^{K / 2} \sum_{\beta} \frac{\overline{a\left(\mathbf{s}_{\beta}^{-}\right)} a\left(\mathbf{s}_{\beta}^{+}\right)}{\sqrt{\left|D^{\beta}\right|}} \exp \left(\frac{i}{\hbar} \mathcal{S}_{\beta}(\mathbf{x})-i \frac{\pi}{2} \mu_{\beta}+i \eta_{\beta} \frac{\pi}{4}\right)+\text { c.c. . }
$$

Under the form 19 it becomes clear that $W_{\mathcal{L}}(\mathbf{x})$ is invariant under symplectic maps. Indeed, such transformations preserve the wedge product, so that all $D_{a b}$ are invariant. As for the action $\mathcal{S}_{\beta}(\mathbf{x})$, it is given by the symplectic area 13 enclosed by a path drawn on the Lagrangian manifold together with the chord joining the two points $\mathbf{x}^{+}$and $\mathbf{x}^{-}$. The 
symplectic area enclosed by a given curve is invariant under any canonical transformation; and for a symplectic map, the chord joining $\mathbf{x}^{+}$to $\mathbf{x}^{-}$is transformed into the chord joining the image of $\mathbf{x}^{+}$and $\mathbf{x}^{-}$. Thus, $(19)$ is invariant under symplectic maps.

However, $W_{\mathcal{L}}(\mathbf{x})$ is a priori not necessarily invariant under generic canonical transformations. Consider first the matrix $D$. Canonical transformations can be characterized by the fact that they preserve the 2 -form $\omega^{2}$ derived from the skew product (10). This 2 -form is defined at each point on the local tangent space, but $\partial \mathbf{x}^{+} / \partial s_{a}^{+}$and $\partial \mathbf{x}^{-} / \partial s_{b}^{-}$in $(18)$ should be considered as belonging to the local tangent space at $\mathbf{x}^{+}$and $\mathbf{x}^{-}$respectively. They will transform as a vector field under a canonical transformation, i.e. along the corresponding linearized maps $M_{+}$and $M_{-}$respectively, so that

$$
D_{a b}=\frac{\partial \mathbf{x}^{-}}{\partial s_{b}^{-}} J \frac{\partial \mathbf{x}^{+}}{\partial s_{a}^{+}} \rightarrow \frac{\partial \mathbf{x}^{-}}{\partial s_{b}^{-}} M_{-}^{T} J M_{+} \frac{\partial \mathbf{x}^{+}}{\partial s_{a}^{+}} .
$$

Since in general $M_{-}^{T} J M_{+} \neq J$ the quantities $D_{a b}$ are not invariant (of course for symplectic maps $M_{+}=M_{-}$and we recover invariance). The same goes for the action $\mathcal{S}_{\beta}(\mathbf{x})$ since for a generic canonical transformation the chord joining $\mathbf{x}^{+}$and $\mathbf{x}^{-}$does not a priori transform into a chord.

This statement being made, we shall however see in section IV that the interference contributions to the mean value Eq. (4) are dominated by short chords, i.e. chords $\boldsymbol{\xi}_{\beta}=$ $\mathbf{x}_{\beta}^{+}-\mathbf{x}_{\beta}^{-}$whose length goes to zero in the semiclassical limit. This means that as $\hbar \rightarrow 0$, the contributions of the Wigner function we will be interested in will be the ones such that $\mathbf{x}_{\beta}^{+} \rightarrow \mathbf{x}_{\beta}^{-}$(although $\mathbf{s}_{\beta}^{+} \not \rightarrow \mathbf{s}_{\beta}^{-}$), see Fig. 3. For these contributions, the distinction between general and linear canonical maps becomes irrelevant. Indeed the tangent spaces near $\mathbf{x}_{\beta}^{+}$ and $\mathbf{x}_{\beta}^{-}$, as well as the linearized maps $M_{+}$and $M_{-}$, can be identified, while the distortion of the chord between $\mathbf{x}_{\beta}^{+}$and $\mathbf{x}_{\beta}^{-}$becomes negligible. In that limit, both the matrix $D^{\beta}$ and the action $\mathcal{S}_{\beta}(\mathbf{x})$ are genuine canonical invariants up to negligible higher-order $\hbar$ corrections. Thus, the contributions $\beta$ which, in the sum (19), are relevant for the calculation of the mean value of smooth operators can be considered as invariant under any (i.e. not necessarily linear) canonical transformation. Interestingly, going back from 19 to 12 , this also means that the same considerations can be applied to this original form of the Wigner function: in the semiclassical limit where only the vicinity of short chords contributes, one can choose arbitrary canonical coordinates to express (12), as all discrepancies will be higher-order $\hbar$ corrections. We will make use of this property in the following section, where we shall allow 
ourselves arbitrary canonical transformations, keeping in mind that this only makes sense in the situation where short chords dominate.

\section{Parametrization invariance of the Wigner function}

The amplitude of $\psi_{\mathcal{L}}(\mathbf{q})$ in $(11)$ is $\mathcal{A}(\mathbf{q})$, which should not depend on the parametrisation $\mathbf{s}$ of the Lagrangian manifold. The Wigner function in $(12)$ is therefore invariant under a change of parameter, and so should be its semiclassical expression (19). This can be checked directly on (19). Indeed, upon a change $\mathbf{s} \mapsto \mathbf{s}^{\prime}, a(\mathbf{s})$ should transform as $\frac{a(\mathbf{s})}{\sqrt{|\partial \mathbf{q} / \partial \mathbf{s}|}}=\frac{a\left(\mathbf{s}^{\prime}\right)}{\sqrt{\left|\partial \mathbf{q} / \partial \mathbf{s}^{\prime}\right|}}$, i.e.

$$
\begin{aligned}
\mathbf{s} & \mapsto \mathbf{s}^{\prime} \\
a(\mathbf{s}) & \mapsto a\left(\mathbf{s}^{\prime}\right)=a(\mathbf{s}) \sqrt{\left|\partial \mathbf{s} / \partial \mathbf{s}^{\prime}\right|}
\end{aligned}
$$

Since using 18 we have

$$
D_{a b}(\mathbf{s}) \mapsto D_{a b}\left(\mathbf{s}^{\prime}\right)=\sum_{i, j=1}^{K} \frac{\partial s_{i}}{\partial s_{a}^{\prime}}\left(\frac{\partial \mathbf{x}^{+}}{\partial s_{i}^{+}} \wedge \frac{\partial \mathbf{x}^{-}}{\partial s_{j}^{-}}\right) \frac{\partial s_{j}}{\partial s_{b}^{\prime}},
$$

the ratio $\overline{a\left(\mathbf{s}^{-}\right)} a\left(\mathbf{s}^{+}\right) / \sqrt{|D|}$ is as expected invariant. The choice of parameter will be guided by convenience. For instance, when considering the eigenstates of classically integrable systems, for which one can define a system of action-angle coordinates, the Lagrangian manifolds of interest are the invariant tori specified by the action variables, and the parameters s can be chosen as the angle variables [28]. In Section IV] we will choose a local parametrization that simplifies calculations for mean values of observables.

\section{Wigner function in the vicinity of the manifold}

The above semiclassical expansion $(19)$ is not valid when $\mathbf{x}$ is in the vicinity of $\mathcal{L}$. Indeed, in the limit $\mathbf{x} \rightarrow \mathcal{L}$ we have $\mathbf{s}^{+} \rightarrow \mathbf{s}^{-}$and $\mathbf{x}^{+} \rightarrow \mathbf{x}^{-}$, and therefore the matrix $D$ becomes singular. The stationary approximation is no longer valid in this case, and we have to start over from 12 .

Let us assume that $\mathcal{L}$ has some curvature only at a classical scale. Keeping only linear terms in the actions $S_{\mathcal{L}}$ in 12 , we get (disregarding the constant phases)

$$
\left.W_{\mathcal{L}}(\mathbf{x})=\frac{1}{(2 \pi \hbar)^{K}} \int d^{K} \mathbf{q}^{\prime} \overline{\mathcal{A}\left(\mathbf{q}-\frac{\mathbf{q}^{\prime}}{2}\right.}\right) \mathcal{A}\left(\mathbf{q}+\frac{\mathbf{q}^{\prime}}{2}\right) e^{\frac{i}{\hbar}\left(\mathbf{p}_{\mathcal{L}}-\mathbf{p}\right) \mathbf{q}^{\prime}}, \quad \text { with } \mathbf{p}_{\mathcal{L}}=\frac{\partial S_{\mathcal{L}}}{\partial \mathbf{q}} .
$$


If the prefactor varies slowly as a function of its arguments, that is, only on a classical scale, then at $\hbar \rightarrow 0$ the amplitudes can be taken out of the integral, so that [29]

$$
W_{\mathcal{L}}(\mathbf{x})=|\mathcal{A}(\mathbf{q})|^{2} \delta\left(\mathbf{p}-\mathbf{p}_{\mathcal{L}}\right)=\int d^{K} \mathbf{q}_{\mathcal{L}}\left|\mathcal{A}\left(\mathbf{q}_{\mathcal{L}}\right)\right|^{2} \delta\left(\mathbf{x}-\mathbf{x}_{\mathcal{L}}\right)=\int d^{K} \mathbf{s}|a(\mathbf{s})|^{2} \delta(\mathbf{x}-\mathbf{x}(\mathbf{s}))
$$

where the last equality comes from the change of variables $\mathbf{q}_{\mathcal{L}} \mapsto \mathbf{s}$, using (22). The righthand side of (25) is again manifestly canonically invariant since, upon integration over an aribtrary test function it will only depend on geometrical properties of $\mathcal{L}$.

At small but finite $\hbar$, taking into account the curvature of the manifold through uniform approximation will lead to an Airy function in the one-dimensional case [25, 30], and to more subtle behaviors in higher dimension [28]. Taking into account such corrections, or the the variation of the prefactor, will in practice broaden the $\delta$ function on the quantum scale. If the Wigner function is convoluted with a classical object, as we shall do below, this will however not affect the final result.

\section{E. Time propagation}

We now introduce a dynamics through some Hamiltonian $\hat{H}$. Let $g_{t}$ be the corresponding classical Hamiltonian flow. For any fixed $t$ it is a canonical transformation (Theorem 4.17 of [5]). Since any canonical transformation maps a Lagrangian manifold into another one (Theorem 4.18 of [5]), the manifold $\mathcal{L}_{t}=g_{t} \mathcal{L}$ obtained by propagation of $\mathcal{L}$ under the classical flow is a Lagrangian manifold.

The strength and beauty of the Maslov association between the Lagrangian manifold $\mathcal{L}$ and the semiclassical wavefunction $\psi_{\mathcal{L}}$ is that the semiclassical propagated wavefunction $\psi_{\mathcal{L}}(t)$ is obtained from the manifold $\mathcal{L}_{t}=g_{t} \mathcal{L}$ following exactly the same procedure. Therefore, the time evolution of the Wigner function $W_{\mathcal{L}}(\mathbf{x})$ is obtained as

$$
W_{\mathcal{L}}(\mathbf{x}, t)=W_{\mathcal{L}_{t}}(\mathbf{x}), \quad \mathcal{L}_{t}=g_{t} \mathcal{L}
$$

This means that the Wigner function at time $t$ is given by Eq. 19 with $\mathcal{L}$ replaced by its time evolution $g_{t} \mathcal{L}$ (and coordinates $\mathbf{s}$ on $g_{t} \mathcal{L}$ are the image of the coordinates $\mathbf{s}$ on $\mathcal{L}$ ). Equation (19) and its time evolved version Eq. 26) will form the basis of our discussion of the mean value of smooth operators. 


\section{EXPECTATION VALUE OF SMOOTH OPERATORS}

\section{A. Stationary point contribution, and why this is not enough}

We now turn to the discussion of the time evolution of the expectation value of an operator $\hat{O}$ for an initial semiclassical wavefunction $\Psi_{0}$ constructed on an initial manifold $\mathcal{L}_{0}$ following the Maslov approach described in section II. From the discussion of section IIIE, the Wigner function $W_{t}(\mathbf{x})$ of the time-evolved wavefunction is given by $W_{\mathcal{L}_{t}}(\mathbf{x})$, expressed as Eq. (19), with $\mathcal{L}_{t}=g_{t} \mathcal{L}_{0}$. The expectation value is then given by Eq. (4).

We will assume that $\hat{O}$ is a classical operator, in the sense that its Wigner transform $O(\mathbf{x})$ behaves like a classical quantity, that is, it has no significant variation on the quantum scale fixed by $\hbar$. This notion that $\hat{O}$ is classical implies that, in the stationary phase approximation of (4), only the phase of $W_{t}(\mathbf{x})$ has to be considered. This phase, appearing in (19), is given by (13) in which the action integrals are taken on the propagated manifold $\mathcal{L}_{t}=$ $g_{t} \mathcal{L}_{0}$. Its derivative with respect to the integration variable $\mathbf{x}$ is given by Eq. (16), namely $\partial \mathcal{S}_{\beta}(\mathbf{x}) / \partial \mathbf{x}=J \boldsymbol{\xi}$, where $\boldsymbol{\xi}$ is the chord joining two points on $\mathcal{L}_{t}$ and having $\mathbf{x}$ as center. Thus the stationary phase condition is given by $\boldsymbol{\xi}=0$. This implies that $\mathbf{x} \in \mathcal{L}_{t}$. The semiclassical expression $(19)$ is actually not valid there, as the prefactor $1 / \sqrt{\left|D^{\beta}\right|}$ diverges. Nevertheless, if one starts from the original expression Eq. (12), the stationary phase condition indeed corresponds to chords of length zero.

This implies obviously that there is no hope to find stationary phase points outside of $\mathcal{L}_{t}$, but also that there are $K$ directions (corresponding to the tangents of $\mathcal{L}_{t}$ at $\mathbf{x}$ ) for which $\boldsymbol{\xi}$ remains zero, and thus half of the eigenvalues of $(\partial \boldsymbol{\xi} / \partial \mathbf{x})$ are zero. As a consequence, one cannot do a simple Gaussian integral to take into account the contribution to (4) of the neighborhood of the stationary phase points. However, in the neighborhood of the manifold one can use the expression (25) for $W_{\mathcal{L}_{t}}(\mathbf{x})$, and the integral (4) then gives

$$
\langle\hat{O}\rangle_{\mathrm{cl}}(t)=\int_{\mathcal{L}_{t}} d^{K} \mathbf{s} O(\mathbf{x}(\mathbf{s}))|a(\mathbf{s})|^{2}
$$

which is interpreted as the classical contribution to the expectation value, again in an explicitly canonically invariant form. Note here that if we accept the form $(27)$ at $t=0$ for $\langle\hat{O}\rangle_{\mathrm{cl}}$, application of (5) immediately yields the form (27) for all times. The classical contribution 27) thus has in some sense the same physical content as the TWA/SCL-IVR approximation (5). 
The fact that at stationary points half of the eigenvalues of $(\partial \boldsymbol{\xi} / \partial \mathbf{x})$ are zero is a sign that the neighborhoods of stationary phase points are rather atypical, which is presumably a first hint of why another kind of contribution needs to be kept here.

Indeed, the essence of the stationary phase approximation is not so much that only stationary phase points contribute (they usually are of measure zero), but that the neighborhood of stationary phase points contains all the points where the phase varies slowly. Indeed, if one considers the integral

$$
\int_{y_{1}}^{y_{2}} d y g(y) \exp \left[\frac{i}{\hbar} f(y)\right]
$$

in the small- $\hbar$ limit, and finds a point $y^{*}$ where $f$ varies slowly, i.e. $f^{\prime}$ is small, then there should be a point in the vicinity of $y^{*}$ where $f^{\prime}$ vanishes. More precisely, if $y^{*}$ is such that $f^{\prime}\left(y^{*}\right)=\epsilon^{*} \ll \hbar\left(y_{2}-y_{1}\right)$, then we can expect that $y_{\text {st }} \simeq y^{*}-\epsilon^{*} / f^{\prime \prime}$, with $f^{\prime \prime}=f^{\prime \prime}\left(y^{*}\right) \simeq$ $f^{\prime \prime}\left(y_{\text {st }}\right)$, is a genuine stationary phase point. Usually, $f^{\prime}(y)$ and $f^{\prime \prime}(y)$ are uncorrelated quantities; and if we vary $y$ until we find a point $y^{*}$ such that $f^{\prime}\left(y^{*}\right)$ is small, there is no reason a priori to expect that at this point $f^{\prime \prime}\left(y^{*}\right)$ is small as well. Thus a priori any point $y^{*}$ where $f^{\prime}\left(y^{*}\right)$ is small should be in the immediate neighborhood of a stationary point $y_{\mathrm{st}}$ where $f^{\prime}\left(y_{\mathrm{st}}\right)=0$. Therefore its contribution to $(28)$ is taken into account as soon as the contribution of the neighborhood of $y_{\text {st }}$ is taken into account (which is what is done in the stationary phase approximation).

In generic cases, the only way to fail to have all the "nearly stationary" points in the neighborhood of an exactly stationary one is to tune a parameter to set precisely to zero the second derivative of the phase. This situation can however be handled by uniform approximation, and can actually be interpreted by saying that the stationary phase point has moved to the complex phase space, but remains nevertheless close.

The situation we consider is significantly different. Indeed here "almost stationary points" are associated with small chords $\boldsymbol{\xi}=\mathbf{x}^{+}\left(\mathbf{s}^{+}\right)-\mathbf{x}^{-}\left(\mathbf{s}^{-}\right)$. The neighborhood of stationary phase points corresponds to the situation where $\mathbf{s}^{+} \simeq \mathbf{s}^{-}$. However it may be that $\mathbf{x}^{+} \simeq \mathbf{x}^{-}$while $\mathbf{s}^{+} \nsucceq \mathbf{s}^{-}$: this situation arises in particular in the case where the endpoints $\mathbf{x}^{+}$and $\mathbf{x}^{-}$of the chord correspond to two distinct regions of the evolved manifold $\mathcal{L}_{t}=g_{t} \mathcal{L}_{0}$. In such a case the geometry of the problem forbids the presence of a neighboring stationary phase point (i.e. with exactly $\boldsymbol{\xi}=0$ ). In the following, we reserve the terminology "small chord" to the ones such that $\mathbf{s}^{+} \not \mathbf{s}^{-}$. The dominant interference contributions to $\langle\hat{O}\rangle(t)$ will come from 
these small chords $\boldsymbol{\xi}$.

The question we may ask is how much we should expect such small chords to actually be present in phase space for the evolved manifold $\mathcal{L}_{t}$. It is clear for instance that if $\mathcal{L}_{0}$ does not specifically display such short chords, we are not expecting them either for rather short time where they should remain non-generic features. However, as time increases, the manifold $\mathcal{L}_{t}$ will generically expand (linearly for integrable systems, but exponentially quickly for chaotic systems), which for bounded system, for which the total phase space volume that can be explored is finite, implies that the typical "distance" between close but distinct sheets of $\mathcal{L}_{t}$ has to go to zero as $t \rightarrow \infty$. Therefore, assuming a small but fixed value of $\hbar$, there should generically be a time at which interference effects associated with short chords will set in.

Let us illustrate this for instance with the case of a one dimensional $(K=1)$ chaotic system characterized by a Lyapunov parameter $\lambda$. In that case we expect that the length of $\mathcal{L}_{t}$ behaves as $l_{0} \exp [\lambda t]$, with $l_{0}$ the length of $\mathcal{L}_{0}$. If the total accessible phase volume $\mathcal{V}$ (that is, the phase-space domain inside which $W_{t}(\mathbf{x})$ is confined) is finite, the typical separation between neighboring sheets of $\mathcal{L}_{t}$ should be $O\left(\left(\mathcal{V} / l_{0}\right) \exp [-\lambda t]\right)$. Small chords, and thus interference effects, will typically set in for times of the order of

$$
t_{\text {s.c. }}=\frac{1}{\lambda} \log \left(\frac{\mathcal{V} l}{\hbar l_{0}}\right)
$$

with $l$ a typical length scale characterizing the Wigner transform $O(\mathbf{x})$ of the observable $\hat{O}$. The time scale $t_{\text {s.c. }}$ is thus essentially the Ehrenfest time for which the "characteristic action" used is the ratio $\mathcal{V} l / \hbar l_{0}$. Being logarithmic in $\hbar$, it is thus a fairly short time.

We therefore consider the contribution to (4) associated with two portions of $\mathcal{L}_{t}$, denoted by $\mathcal{L}_{+}$and $\mathcal{L}_{-}$and locally parameterized by $\mathbf{s}^{+}$and $\mathbf{s}^{-}$respectively, which come close to one another. The corresponding interference contribution to the expectation value is an integral of the form

$$
\int d^{K} \mathbf{x} \mathfrak{A}(\mathbf{x}) e^{\frac{i}{\hbar} \mathcal{S}(\mathbf{x})},
$$

where, dropping indices $f, \beta$ and $\mathcal{L}$, the action $\mathcal{S}$ is given by (13) and the smoothly varying prefactor by

$$
\mathfrak{A}(\mathbf{x}) \equiv\left(\frac{2}{\pi \hbar}\right)^{K / 2} \frac{\overline{a\left(\mathbf{s}^{-}\right)} a\left(\mathbf{s}^{+}\right) e^{i \eta \pi / 4-i \mu \pi / 2}}{\sqrt{|D|}} O(\mathbf{x}),
$$

with $\mathbf{s}^{+}, \mathbf{s}^{-}, D, \eta$ and $\mu$ associated with the different chords $\boldsymbol{\xi}$ joining $\mathcal{L}_{+}$and $\mathcal{L}_{-}$and going through $\mathbf{x}$, as in Eq. 19.). 


\section{B. One-dimensional case}

Our goal in this subsection is to perform the phase space integral in Eq. (30) in the one-dimensional case $K=1$. We place ourselves in the context of a chaotic system, where for time $t$ larger than the characteristic time $t_{\text {s.c. }}$ defined in $(29)$, the various sheets of the manifold $\mathcal{L}_{t}$ will tend to align along the manifold, and therefore locally be oriented along essentially the same direction. In this picture, there will be a "parallel" direction (along the general direction of both considered sheets of $\mathcal{L}_{t}$ ) along which quantities vary only on a classical scale, and a "transverse" one, where variations are on the quantum scale (see Fig. 3 for illustration).

We shall see that the integration in the transverse direction, which is associated with rapid variations of the phase away from the middle of the two sheets, can be done analytically using stationary phase approximation. The stationary phase approximation on the other hand cannot be applied for structural reasons to the integral along the parallel direction, which corresponds to a much weaker (almost the absence of) variation of the phase and is much more idiosyncratic, and we shall discuss a couple of limiting cases.

\section{Canonical change of variables}

As discussed in section IIIB, when, as is the case here, only short chords contributions are taken into account, the expression of the semiclassical Wigner function (19), and thus the integral (30), is invariant under an arbitrary canonical change of variable. We therefore introduce a pair of canonical variables $x=\left(x_{\|}, x_{\perp}\right)$ (see Fig. 3), for which the integral (30) simplifies. To do this, let $\mathcal{L}_{c}$ be any smooth curve lying inbetween $\mathcal{L}_{+}$and $\mathcal{L}_{-}$, and $\lambda_{0}$ some coordinate on $\mathcal{L}_{c}$. We note $x_{0}=x\left(\lambda_{0}\right)$ the phase point on $\mathcal{L}_{c}$ with coordinate $\lambda_{0}$. At each point $x_{0} \in \mathcal{L}_{c}$ the corresponding chord $\xi\left(x_{0}\right)$ defines a direction in phase space. Any point $x$ in the region inbetween $\mathcal{L}_{+}$and $\mathcal{L}_{-}$is of the form $x_{0}+u \xi\left(x_{0}\right)$ for some $x_{0} \in \mathcal{L}_{c}$ and $u \in\left[-\frac{1}{2}, \frac{1}{2}\right]$. One can therefore adopt a local coordinate system such that $x=\left(x_{\|}, x_{\perp}\right)$ with $x_{\|}=\lambda_{0}$ the coordinate of $x$ along $\mathcal{L}_{c}$ and $x_{\perp} \propto u$ the coordinate along the chord $\xi\left(x_{0}\right)$. We choose $x_{\perp}$ so that $d x_{\|} \wedge d x_{\perp}=d p \wedge d q$, which makes the change of variables from $(p, q)$ to $\left(x_{\|}, x_{\perp}\right)$ canonical.

In such a coordinate system, $\mathcal{L}_{c}$ is characterized by the equation $x_{\perp}=0$. Moreover, if 


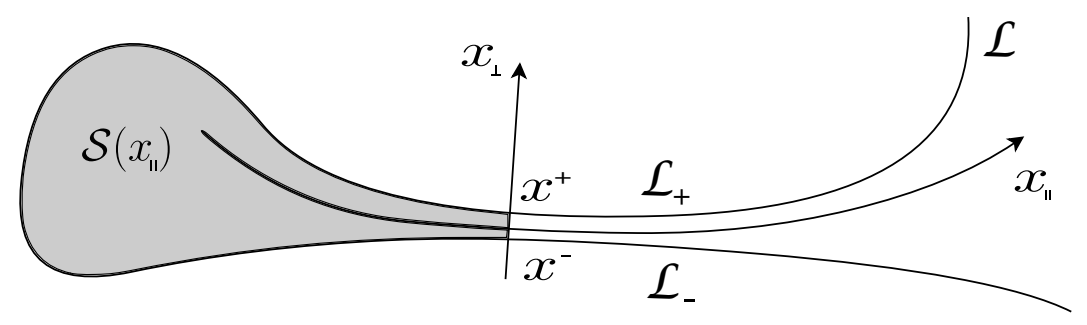

FIG. 3. Local system of coordinates used for evaluation of Eq. (30) for small chords. The coordinate $x_{\|}$is the parallel direction which follow the (almost identical on the classical scale) pieces $\mathcal{L}_{+}$and $\mathcal{L}_{-}$of the Lagrangian manifold $\mathcal{L} ; x_{\perp}$ is the transverse direction.

one considers a chord $\xi$ joining $\mathcal{L}_{+}$and $\mathcal{L}_{-}$and centered at a point of $\mathcal{L}_{c}, \xi$ is by definition oriented in the transverse direction, implying that its parallel component vanishes, namely $\xi_{\|}=0$. The endpoints $x^{+}$and $x^{-}$of the chord $\xi\left(x_{0}\right)$ going through a point $x_{0}=\left(x_{\|}, 0\right)$ are defined by $\frac{1}{2}\left(x^{+}+x^{-}\right)=x_{0}=\left(x_{\|}, 0\right)$ and $\xi=x^{+}-x^{-}$, and therefore we have

$$
x^{ \pm}=\left(x_{\|}, \pm \frac{1}{2} w\left(x_{\|}\right)\right)
$$

with $w\left(x_{\|}\right)=|\xi|$. Therefore, in the units specified by the measure $d x_{\perp}$, the function $w\left(x_{\|}\right)$ gives the width of the region of small chord lengths at point $x_{0}$.

\section{Semiclassical integration in the transverse direction}

We are now in a position to perform the integral (30) in the transverse direction, for a fixed value of $x_{\|}$. As $\partial \mathcal{S} / \partial x=J \xi$, we have $\partial \mathcal{S} / \partial x_{\perp}=\xi_{\|}$, so that the stationary condition $\partial \mathcal{S} / \partial x_{\perp}=0$ is given by $\xi_{\|}=0$, that is, stationary points lie on $\mathcal{L}_{c}$.

Fixing a point $x_{0}=\left(x_{\|}, 0\right)$ on $\mathcal{L}_{c}$, we can perform the integral along $x_{\perp}$ in the stationary phase approximation. By construction the stationary point is $x_{\perp}=0$, and Gaussian integration yields

$$
\int_{-\infty}^{\infty} d x_{\perp} e^{\frac{i}{2 \hbar}\left(\frac{\partial \xi_{\|}}{\partial x_{\perp}}\right)_{x_{\perp}=0} x_{\perp}^{2}}=\sqrt{\frac{2 i \pi \hbar}{\left(\partial \xi_{\|} / \partial x_{\perp}\right)_{x_{\perp}=0}}} .
$$

Let us now compute the derivative $\left(\partial \xi_{\|} / \partial x_{\perp}\right)_{x_{\perp}=0}$. At an arbitrary point $x$ in the neighborhood of $x_{0}$, the endpoints $x^{+}(x)$ and $x^{-}(x)$ of the chord $\xi(x)$ going through $x$ coincide with endpoints of chords going through points lying on $\mathcal{L}_{c}$. More precisely, there exists some $\lambda_{+} \in \mathcal{L}_{c}$ such that $x^{+}(x)=x^{+}\left(\lambda_{+}, 0\right)$, and some $\lambda_{-} \in \mathcal{L}_{c}$ such that $x^{-}(x)=x^{-}\left(\lambda_{-}, 0\right)$. As 
a consequence, Eq. (32) yields

$$
x=\frac{x^{+}(x)+x^{-}(x)}{2}=\frac{x^{+}\left(\lambda_{+}, 0\right)+x^{-}\left(\lambda_{-}, 0\right)}{2}=\left(\frac{\lambda_{+}+\lambda_{-}}{2}, \frac{w\left(\lambda_{+}\right)-w\left(\lambda_{-}\right)}{4}\right),
$$

and the corresponding chord $\xi$ reads

$$
\xi(x)=x^{+}(x)-x^{-}(x)=\left(\lambda_{+}-\lambda_{-}, \frac{w\left(\lambda_{+}\right)+w\left(\lambda_{-}\right)}{2}\right) .
$$

Since both $\lambda_{+}$and $\lambda_{-}$are close to $x_{0}$ we can expand to first order

$$
x_{\perp}=\frac{w\left(\lambda_{+}\right)-w\left(\lambda_{-}\right)}{4} \simeq \frac{\lambda_{+}-\lambda_{-}}{4} w^{\prime}\left(x_{\|}\right)=\frac{\xi_{\|}}{4} w^{\prime}\left(x_{\|}\right)
$$

which yields

$$
\left(\frac{\partial \xi_{\|}}{\partial x_{\perp}}\right)_{x_{\perp}=0}=\frac{4}{w^{\prime}\left(x_{\|}\right)} .
$$

We thus obtain for the result of the transverse integration at fixed $x_{\|}$

$$
\int d x_{\perp} \mathfrak{A}(x) e^{\frac{i}{\hbar} \mathcal{S}(x)}=\frac{1}{2} \mathfrak{A}\left(x_{\|}, 0\right) e^{\frac{i}{\hbar} \mathcal{S}\left(x_{\|}, 0\right)} \sqrt{2 \pi \hbar\left|w^{\prime}\left(x_{\|}\right)\right|} e^{i \frac{\pi}{4} \operatorname{sgn}\left(w^{\prime}\left(x_{\|}\right)\right)}
$$

with $\mathfrak{A}$ defined by Eq. (31).

\section{Integration in the parallel direction}

To proceed with the last integral, one should bear in mind that the parameterization of the Lagrangian manifold $\mathcal{L}_{t}$ is essentially arbitrary, and that any parametrization $\mathbf{s}^{\prime}$ of $\mathcal{L}_{t}$ can be used as long as $a(\mathbf{s})$ transforms into $a\left(\mathbf{s}^{\prime}\right)$ according to 22 .

Therefore, instead of using parameters $s^{+}$and $s^{-}$in the regions around $x^{+}$and $x^{-}$we can use $x_{\|}$as a parameter for both, with $x_{\|}$the coordinate on $\mathcal{L}_{c}$ such that the endpoints of the chord going through $\left(x_{\|}, 0\right)$ coincide with $x^{+}$and $x^{-}$(note that $x_{\|}$coincides with $\lambda_{+}$and $\lambda_{-}$introduced above for $s^{+}$and $s^{-}$respectively). We shall denote by $a_{+}$and $a_{-}$the amplitudes in the regions around $x^{+}$and $x^{-}$, so that $a_{+}\left(x_{\|}\right) \equiv a\left(s^{+}\right) \sqrt{\left|d s^{+} / d x_{\|}\right|}$ and $a_{-}\left(x_{\|}\right) \equiv a\left(s^{-}\right) \sqrt{\left|d s^{+} / d x_{\|}\right|}$. With that parametrization the prefactor in Eq. (19) is

proportional to $\overline{a_{-}\left(x_{\|}\right)} a_{+}\left(x_{\|}\right) / \sqrt{\left|D_{\|}\right|}$with $D_{\|}$expressed by 17 but in the new variables, that is,

$$
D_{\|}=\left(\frac{\partial x_{\|}^{+}}{\partial x_{\|}}\right)^{T}\left(\frac{\partial x_{\perp}^{-}}{\partial x_{\|}}\right)-\left(\frac{\partial x_{\perp}^{+}}{\partial x_{\|}}\right)^{T}\left(\frac{\partial x_{\|}^{-}}{\partial x_{\|}}\right)
$$


Here, $x^{ \pm}=\left(x_{\|}^{ \pm}, x_{\perp}^{ \pm}\right)$is given by Eq. (32), so that $\partial x^{ \pm} / \partial x_{\|}=\left(1, \pm \frac{1}{2} w^{\prime}\left(x_{\|}\right)\right)$, which inserted in $(39)$ yields $D_{\|}=w^{\prime}\left(x_{\|}\right)$. Introducing the notation $O\left(x_{\|}\right) \equiv O\left(x\left(x_{\|}, 0\right)\right)$ and $\mathfrak{A}\left(x_{\|}\right) \equiv$ $\mathfrak{A}\left(x\left(x_{\|}, 0\right)\right)$ (and for later use $\left.\mathcal{S}\left(x_{\|}\right) \equiv \mathcal{S}\left(x\left(x_{\|}, 0\right)\right)\right)$ we thus have

$$
\mathfrak{A}\left(x_{\|}\right)=\sqrt{\frac{2}{\pi \hbar}} \frac{\overline{a_{-}\left(x_{\|}\right)} a_{+}\left(x_{\|}\right) e^{i \eta \pi / 4-i \mu \pi / 2}}{\sqrt{\left|w^{\prime}\left(x_{\|}\right)\right|}} O\left(x_{\|}\right) .
$$

The factor $1 / \sqrt{\left|w^{\prime}\left(x_{\|}\right)\right|}$in 40 thus exactly cancels the $\sqrt{\left|w^{\prime}\left(x_{\|}\right)\right|}$in (38) originating from the transverse integral. Taking into account the summation over all possible chords labeled by $\beta$ we finally get

$$
\langle\hat{O}\rangle=\langle\hat{O}\rangle_{\text {classic }}+\sum_{\beta=\text { short chord }} \int d x_{\|} O\left(x_{\|}\right) \overline{a_{-}^{\beta}\left(x_{\|}\right)} a_{+}^{\beta}\left(x_{\|}\right) e^{\frac{i}{\hbar} \mathcal{S}_{\beta}\left(x_{\|}\right)-i \mu_{\beta} \frac{\pi}{2}+i \eta_{\beta} \frac{\pi}{4}+i \eta_{\beta} \frac{\pi}{4}}+\text { c.c. , }
$$

where $\mu_{\beta}$ is the Maslov index of any path joining $\mathcal{L}_{+}^{\beta}$ to $\mathcal{L}_{-}^{\beta}$ and $\eta^{\perp}$ is the sign of $w^{\prime}\left(x_{\|}\right)$.

Similarly to the classical contribution Eq. (27), the remaining integration over $x_{\|}$is actually very much classical-like. Indeed, because only phases that vary extremely slowly contribute, all factors in the integrand vary only on the classical scale, including the exponential term which contains an $\hbar$ in its exponent. This is therefore a rather atypical integral in the context of semiclassical physics : it cannot be evaluated using the separation of scales typical of the stationary phase approximation (where anything varying only on the classical scale can be assumed frozen in the relevant range of integration), which actually is the reason why a blind application of stationary phase approximation fails for the calculation of the expectation value of operators.

\section{Generalization to dimension $K$}

If we look back at the physical meaning of the calculation leading to Eq. (41), it can be summarized as follow. First, we have identified that the origin of the intereference terms corresponds to pairs of sheets of the Lagrangian manifold that, under the classical time evolution, become close to each other on the quantum scale. On the classical scale they can therefore be considered as essentially identical as $\hbar \rightarrow 0$, and one can therefore parametrize both of them by the same coordinate $\mathbf{x}_{\|}$. Introducing then a transverse direction $\mathbf{x}_{\perp}$ such that $\left(\mathbf{x}_{\|}, \mathbf{x}_{\perp}\right)$ forms a canonical pair of variable, the integral along the transverse direction can be done using stationary phase approximation, while the one on the parallel direction does not involve any rapidly oscillating phase and is thus essentially of a classical nature. 
What eventually makes this calculation tractable is that we can apply a canonical change of variable at various steps of the derivation and place ourselves in the coordinate system for which the computation is the simplest. This is possible because, as dicussed in section IIIB, the relevant contribution to the problem we are considering are invariant under canonical changes of variable.

Having done this carefully for the one-dimensional case, we shall see now that the result (41) can be obtained more directly and extended to arbitrary dimension if we start from the very definition (3) of the Wigner function, with $\Psi_{t}$ the semiclassical wavefunction constructed from the propagated Lagrangian manifold $\mathcal{L}_{t}$, and choose the right coordinate system. Again, as follows from the discussion of Section IIIB, the relevant contributions to (4) of (3) can, too, be considered as canonical invariants, which allows us to choose freely the coordinate system.

Consider thus a contribution to the interference terms associated with a pair of sheets $\left(\mathcal{L}^{+}, \mathcal{L}^{-}\right)$of $\mathcal{L}$ which come extremely close to each other. On the classical scale these two sheets can be viewed as identical, and because they are Lagrangian it is always possible to find a system of canonical coordinates $\left(\mathbf{x}_{\|}, \mathbf{x}_{\perp}\right.$ ) such that $\mathbf{x}_{\|}$parametrizes $\mathcal{L}^{ \pm}$(in the sense that $\left(\mathbf{x}_{\|}, 0\right) \in \mathcal{L}^{ \pm}$if we do not distinguish between $\mathcal{L}^{+}$and $\mathcal{L}^{-}$) and $\mathbf{x}_{\perp}$ is transverse to it. Here however, and contrarily to the approach we used in section IVB for the onedimensional case, we use a coordinate system $\left(\mathbf{x}_{\|}, \hat{\mathbf{x}}_{\perp}\right)$ such that $\hat{\mathbf{x}}_{\perp}$ can, too, be used as a parametrization of $\mathcal{L}$, so that it can play the role of $\mathbf{q}$ in Eq. (3). A simple choice of coordinates for such a requirement is the sheer $\hat{\mathbf{x}}_{\perp} \equiv \mathbf{x}_{\perp}+\mu \mathbf{x}_{\|}$(with $\mu \neq 0$ arbitrary). We use from now on the variables $\left(\mathbf{x}_{\|}, \hat{\mathbf{x}}_{\perp}\right)$.

In this coordinate system, the integral (4) reads

$\frac{1}{(2 \pi \hbar)^{K}} \iiint d^{K} \mathbf{x}_{\|} d^{K} \hat{\mathbf{x}}_{\perp} d^{K} \hat{\mathbf{x}}_{\perp}^{\prime} \mathcal{A}\left(\hat{\mathbf{x}}_{\perp}+\frac{\hat{\mathbf{x}}_{\perp}^{\prime}}{2} \overline{\mathcal{A}\left(\hat{\mathbf{x}}_{\perp}-\frac{\hat{\mathbf{x}}_{\perp}^{\prime}}{2}\right)} O\left(\mathbf{x}_{\|}, \hat{\mathbf{x}}_{\perp}\right) e^{-\frac{i}{\hbar} \mathbf{x}_{\|} \cdot \hat{\mathbf{x}}_{\perp}^{\prime}+\frac{i}{\hbar} S\left(\hat{\mathbf{x}}_{\perp}+\frac{\hat{\mathbf{x}}_{\perp}^{\prime}}{2}\right)-\frac{i}{\hbar} S\left(\hat{\mathbf{x}}_{\perp}-\frac{\hat{\mathbf{x}}_{\perp}^{\prime}}{2}\right)}\right.$,

where $O\left(\mathbf{x}_{\|}, \hat{\mathbf{x}}_{\perp}\right) \equiv O\left(\mathbf{x}\left(\mathbf{x}_{\|}, \hat{\mathbf{x}}_{\perp}\right)\right)$. Changing variables in the integral (42) from $\hat{\mathbf{x}}_{\perp}, \hat{\mathbf{x}}_{\perp}^{\prime}$ to $\hat{\mathbf{x}}_{\perp}^{+}$, $\hat{\mathbf{x}}_{\perp}^{-}$with $\hat{\mathbf{x}}_{\perp}^{ \pm}=\hat{\mathbf{x}}_{\perp} \pm \frac{1}{2} \hat{\mathbf{x}}_{\perp}^{\prime}$ (with Jacobian equal to 1), Eq. (42) gives

$$
\frac{1}{(2 \pi \hbar)^{K}} \iiint d^{K} \mathbf{x}_{\|} d^{K} \hat{\mathbf{x}}_{\perp}^{+} d^{K} \hat{\mathbf{x}}_{\perp}^{-} \mathcal{A}\left(\hat{\mathbf{x}}_{\perp}^{+}\right) \overline{\mathcal{A}\left(\hat{\mathbf{x}}_{\perp}^{-}\right)} O\left(\mathbf{x}_{\|}, \frac{\hat{\mathbf{x}}_{\perp}^{+}+\hat{\mathbf{x}}_{\perp}^{-}}{2}\right) e^{-\frac{i}{\hbar} \mathbf{x}_{\|} \cdot\left(\hat{\mathbf{x}}_{\perp}^{+}-\hat{\mathbf{x}}_{\perp}^{-}\right)+\frac{i}{\hbar} S\left(\hat{\mathbf{x}}_{\perp}^{+}\right)-\frac{i}{\hbar} S\left(\hat{\mathbf{x}}_{\perp}^{-}\right)} .
$$

We then use the fact that $\mathcal{L}$ can be parametrized locally by an arbitrary parameter $\mathbf{s}$ and that the amplitude $\mathcal{A}\left(\hat{\mathbf{x}}_{\perp}\right)$ of the semiclassical wavefunction is related to the density $a(\mathbf{s})$ 
on $\mathcal{L}$ by $\mathcal{A}\left(\hat{\mathbf{x}}_{\perp}\right)=a(\mathbf{s}) / \sqrt{\left|\partial \hat{\mathbf{x}}_{\perp} / \partial \mathbf{s}\right|}$. Choosing $\mathbf{s}=\mathbf{x}_{\|}$as a local parameter in the vicinity of points $\mathbf{x}^{+}$and $\mathbf{x}^{-}$we have

$$
\mathcal{A}\left(\hat{\mathbf{x}}_{\perp}^{+}\right)=\frac{a\left(\mathbf{x}_{\|}^{+}\right)}{\sqrt{\left|\partial \hat{\mathbf{x}}_{\perp}^{+} / \partial \mathbf{x}_{\|}^{+}\right|}}, \quad \mathcal{A}\left(\hat{\mathbf{x}}_{\perp}^{-}\right)=\frac{a\left(\mathbf{x}_{\|}^{-}\right)}{\sqrt{\mid \partial \hat{\mathbf{x}}_{\perp}^{-} / \partial \mathbf{x}_{\|}^{-1}}} .
$$

We now perform the stationary phase integration over the two variables $\hat{\mathbf{x}}_{\perp}^{+}$and $\hat{\mathbf{x}}_{\perp}^{-}$in 43 . The critical points $\left(\hat{\mathbf{x}}_{\perp}^{+*}, \hat{\mathbf{x}}_{\perp}^{-*}\right)$ are those where the gradient of the phase vanishes, yielding the two conditions $\mathbf{x}_{\|}{ }^{+}\left(\hat{\mathbf{x}}_{\perp}^{+*}\right)=\mathbf{x}_{\|}$and $\mathbf{x}_{\|}{ }^{-}\left(\hat{\mathbf{x}}_{\perp}^{-*}\right)=\mathbf{x}_{\|}$, corresponding respectively to the points on $\mathcal{L}^{+}$and $\mathcal{L}^{-}$parameterized by $\mathbf{x}_{\|}$. The Hessian matrix is a block-diagonal matrix with blocks given by $\left(\partial \mathbf{x}_{\|}^{+} / \partial \hat{\mathbf{x}}_{\perp}^{+}\right)$and $\left(\partial \mathbf{x}_{\|}^{-} / \partial \hat{\mathbf{x}}_{\perp}^{-}\right)$. Gaussian integration thus gives an amplitude

$$
(2 \pi \hbar)^{K} \frac{1}{\sqrt{\left|\left(\frac{\partial \mathbf{x}_{\|}^{+}}{\partial \hat{\mathbf{x}}_{\perp}^{+}}\right)\left(\frac{\partial \mathbf{x}_{\|}^{-}}{\partial \hat{\mathbf{x}}_{\perp}^{-}}\right)\right|}} .
$$

Inserting (44) and (45) into Eq. (43) at the stationary point $\mathbf{x}_{\|}{ }^{+}=\mathbf{x}_{\|}{ }^{-}=\mathbf{x}_{\|}$, we get

$$
\langle\hat{O}\rangle=\langle\hat{O}\rangle_{\text {classic }}+\sum_{\beta=\text { short chord }} \int d^{K} \mathbf{x}_{\|} O\left(\mathbf{x}_{\|}\right) \overline{a_{-}^{\beta}\left(\mathbf{x}_{\|}\right)} a_{+}^{\beta}\left(\mathbf{x}_{\|}\right) e^{\frac{i}{\hbar} \mathcal{S}_{\beta}\left(\mathbf{x}_{\|}\right)-i \theta_{\beta} \frac{\pi}{4}}+\text { c.c. }
$$

where (as in Sect. III A) each chord corresponds to two stationary points for which the roles of $\hat{\mathbf{x}}_{\perp}^{+*}$ and $\hat{\mathbf{x}}_{\perp}^{-*}$ are exchanged, yielding two complex conjugated terms. In this equation,

$$
O_{\beta}\left(\mathbf{x}_{\|}\right) \equiv O_{\beta}\left(\mathbf{x}_{\|}, \frac{\hat{\mathbf{x}}_{\perp}^{+}\left(\mathbf{x}_{\|}\right)+\hat{\mathbf{x}}_{\perp}^{-}\left(\mathbf{x}_{\|}\right)}{2}\right)
$$

is the Wigner transform of the operator $\hat{O}$ evaluated in $\mathbf{x}_{\|}$at the mid-point between $\mathcal{L}^{+}$and $\mathcal{L}^{-}$

$$
\mathcal{S}_{\beta}\left(\mathbf{x}_{\|}\right) \equiv \mathcal{S}_{\beta}\left(\mathbf{x}_{\|}, \frac{\hat{\mathbf{x}}_{\perp}^{+}\left(\mathbf{x}_{\|}\right)+\hat{\mathbf{x}}_{\perp}^{-}\left(\mathbf{x}_{\|}\right)}{2}\right)
$$

is the action Eq. (13) evaluated at the same location, i.e. the action integral on the contour formed by a path on $\mathcal{L}$ from $\mathbf{x}_{\beta}^{+}$to $\mathbf{x}_{\beta}^{-}$which is closed by a straight line between $\mathbf{x}_{\beta}^{-}$and $\mathbf{x}_{\beta}^{+}$; and $\theta_{\beta}$ is a global index. With this parameterization, the fact that the remaining integral on $\mathbf{x}_{\|}$cannot be performed within a stationary phase approximation approach becomes obvious. For $K=1$ this expression coincides with Eq. 41.

The index $\theta_{\beta}$ in 46 results from a contribution from the double Gaussian integration (45) over $\hat{\mathbf{x}}_{\perp}^{+}, \hat{\mathbf{x}}_{\perp}^{-}$performed at fixed $\mathbf{x}_{\|}$(see Fig. 4), and from Maslov indices appearing in the definition of the semiclassical wavefunction constructed on the Lagrangian manifold $\mathcal{L}_{t}$. The stationary phase condition in the Gaussian integration is met when points $\mathbf{x}^{+}$and $\mathbf{x}^{-}$ 


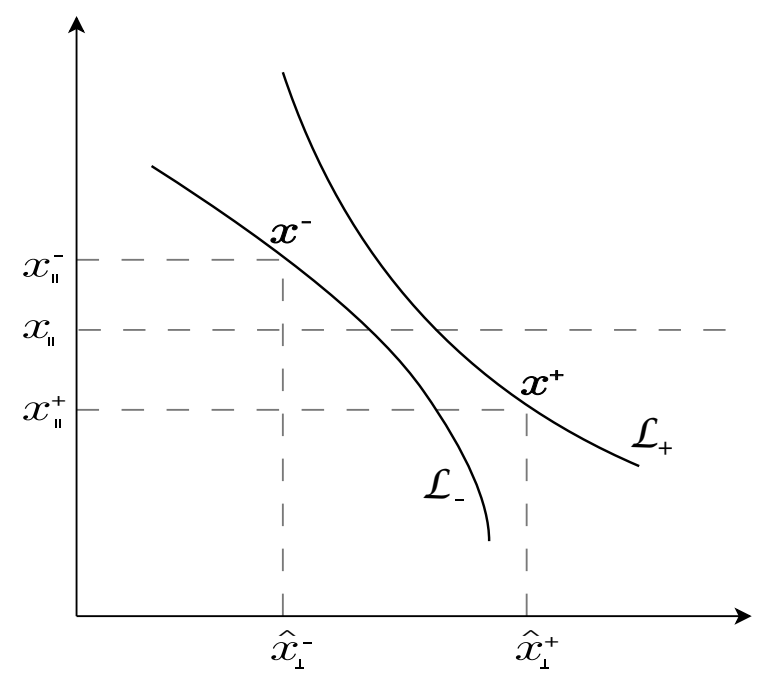

FIG. 4. Parametrization used for the integral (42). The integration variables are $\left(\hat{\mathbf{x}}_{\perp}^{+}, \hat{\mathbf{x}}_{\perp}^{-}, \mathbf{x}_{\|}\right)$, which all can be used (locally) as parametrization of $\mathcal{L}_{ \pm}$. The stationary phase conditions reads $\mathbf{x}_{\|}{ }^{+}\left(\hat{\mathbf{x}}_{\perp}^{+}\right)=\mathbf{x}_{\|}=\mathbf{x}_{\|}^{-}\left(\hat{\mathbf{x}}_{\perp}^{-}\right)$.

are such that $\mathbf{x}_{\|}^{+}=\mathbf{x}_{\|}{ }^{-}=\mathbf{x}_{\|}$. At that point, since $\mathcal{L}_{+}$and $\mathcal{L}_{-}$are almost parallel, the two blocks $\left(\partial \mathbf{x}_{\|}{ }^{+} / \partial \hat{\mathbf{x}}_{\perp}^{+}\right)$and $\left(\partial \mathbf{x}_{\|}{ }^{-} / \partial \hat{\mathbf{x}}_{\perp}^{-}\right)$of the Hessian matrix behave in the same way, so that the phase arising from Gaussian integration is zero. The total phase contribution in (46) thus reduces to the Maslov indices of the wavefunctions at the stationary phase points, i.e. $\theta_{\beta}=\mu_{\beta}$.

\section{Discussion}

In general, the specific characteristics of the initial density $a(\mathbf{s})$, the shape of the evolved manifold $\mathcal{L}_{t}$, and the specific form of the Wigner transform $O(\mathbf{x})$ of the considered operator, all affect the final result, and it is not possible to provide an expression for the last integral on $\mathbf{x}_{\|}$that would be valid on a completely general basis. In many circumstances, one would have to resort to numerical integration to perform this last step. On the other hand, and for the same reason stationary phase cannot be applied, this is a "simple" integral which, when computed numerically, does not require a particularly fine grid to achieve good precision.

Going back to $K=1$, further progress can be made however in two limiting cases that we discuss further now. The first one corresponds to times where the first interference contributions start to appear (i.e. $t \simeq t_{\text {s.c. }}$ ). In that case, short chords are not yet a typical 
feature of the evolved Lagrangian manifold, and will generically correspond to a bottleneck near a point $\mathbf{x}_{0}$ where the two sheets are closest. In that case one recovers the rather typical situation where it is the variation of the phase $\mathcal{S}\left(x_{\|}\right)$which cuts off the integral on $x_{\|}$. Using the fact that $\partial \mathcal{S} / \partial x_{\|}=\xi_{\perp}$, and expanding up to order 3 we get

$$
\mathcal{S}\left(x_{\|}\right)=\mathcal{S}\left(x_{0}\right)+w_{0}\left(x_{\|}-x_{0}\right)+\frac{w_{0}^{\prime \prime}}{6}\left(x_{\|}-x_{0}\right)^{3},
$$

since at the bottleneck point $\mathbf{x}_{0}$ the length $w$ reaches a minimum and thus $w_{0}^{\prime}=0$. If we take the convention that $w^{\prime}(0)>0$ for $x_{\|}>0$ we then have

$$
\int_{-\infty}^{\infty} d x_{\|} e^{\frac{i}{\hbar}\left(w(0) x_{\|}+\frac{w^{\prime \prime}(0)}{6} x_{\|}^{3}\right)}=\left(\frac{2 \hbar}{w_{0}^{\prime \prime}}\right)^{1 / 3} \mathrm{Ai}\left[w_{0}\left(\frac{2 \hbar}{w_{0}^{\prime \prime}}\right)^{1 / 3}\right]
$$

and the integral in 41 reduces to

$$
\langle\hat{O}\rangle_{\beta}=a_{+}^{\beta}\left(x_{0}\right) a_{-}^{\beta}\left(x_{0}\right) O\left(x_{0}\right) e^{-i \mu_{\beta} \frac{\pi}{2}+i \eta_{\beta} \frac{\pi}{4}+i \eta_{\beta}^{\perp} \frac{\pi}{4}}\left(\frac{2 \hbar}{w_{0}^{\prime \prime \beta}}\right)^{1 / 3} \mathrm{Ai}\left[w_{0}^{\beta}\left(\frac{2 \hbar}{w_{0}^{\prime \prime}{ }_{0}^{\beta}}\right)^{1 / 3}\right] .
$$

The second limiting case we shall consider will on the other hand correspond to the long times limit $t \gg t_{\text {s.c. }}$ of a chaotic dynamics, for which we further assume that the region $\operatorname{supp}(O)$ inside which $O(\mathbf{x})$ is significant, although large on the quantum scale, remains relatively small on the classical one, and in particular on the scale on which the stable and unstable manifolds of the classical motion change significantly their shape. In that case, because $t \gg t_{\text {s.c. }}$, we can assume that the various pieces of the evolved Lagrangian manifold $\mathcal{L}_{t}$, and in particular $\mathcal{L}_{+}$and $\mathcal{L}_{-}$, essentially align on the unstable manifold of the dynamics, and therefore remain essentially parallel when they go through $\operatorname{supp}(O)$. Furthermore, because of the exponential stretching along the unstable manifold, the points of $\mathcal{L}_{ \pm} \cap \operatorname{supp}(O)$ all originate from a very narrow neighborhood of the original manifold $\mathcal{L}_{0}$, and we can assume $a_{ \pm}\left(x_{\|}\right)=a_{ \pm}=$const. In that case, Eq. (41) reduces to

$$
\langle\hat{O}\rangle_{\beta}=\overline{a_{-}^{\beta}} a_{+}^{\beta} e^{\frac{i}{\hbar} \mathcal{S}_{\beta}\left(x_{0}\right)-\mu \frac{\pi}{2}} \tilde{O}\left(w_{\beta} / \hbar\right)
$$

(with $w_{\beta}$ the (constant) spacing between $\mathcal{L}_{+}^{\beta}$ and $\mathcal{L}_{-}^{\beta}$ ), and is thus expressed in terms of the Fourier component

$$
\tilde{O}\left(w_{\beta} / \hbar\right)=\int d x_{\|} O\left(x_{\|}\right) e^{\frac{i}{\hbar} w_{\beta}\left(x_{\|}-x_{0}\right)}
$$

of $O\left(x_{\|}\right)$, i.e. of a cut of $O(x)$ along an unstable manifold. Note that a rescaling of $x_{\|}$would not change that result. 


\section{CONCLUSION}

We have obtained a semiclassical expression for the mean value of operators as a sum of a classical contribution

$$
\langle\hat{O}\rangle_{\mathrm{cl}}(t)=\int d \mathbf{x} W_{0}(\mathbf{x}) O\left(g_{t} \mathbf{x}\right)
$$

corresponding to the TWA/LSC-IVR, and oscillatory terms associated with short chords. The final expression reads

$$
\langle\hat{O}\rangle=\langle\hat{O}\rangle_{\mathrm{cl}}(t)+\sum_{\beta} \int d \mathbf{x}_{\|} O\left(\mathbf{x}_{\|} \overline{a_{-}^{\beta}\left(\mathbf{x}_{\|}\right)} a_{+}^{\beta}\left(\mathbf{x}_{\|}\right) e^{\frac{i}{\hbar} \mathcal{S}_{\beta}\left(\mathbf{x}_{\|}\right)-i \mu_{\beta} \frac{\pi}{4}}+\right.\text { c.c. }
$$

with $\mathcal{S}_{\beta}\left(\mathbf{x}_{\|}\right)$the action (13) evaluated at the mid-point between $\mathbf{x}^{+}\left(\mathbf{x}_{\|}\right)$and $\mathbf{x}^{-}\left(\mathbf{x}_{\|}\right)$(the points with parameter $\mathbf{x}_{\|}$on the sheets $\mathcal{L}^{+}$and $\mathcal{L}^{-}$of $\left.\mathcal{L}\right), O\left(\mathbf{x}_{\|}\right)$the value of the Wigner transform of the operator $\hat{O}$ at the same location, and $\mu_{\beta}$ the Maslov index of the path joining $\mathcal{L}^{+}$and $\mathcal{L}^{-}$on which $\mathcal{S}$ is computed. Here, we have used the two invariances of the semiclassical Wigner function, invariance under canonical change of variables (again in the approximation where only short chords contribute) and invariance under parametrization of $\mathcal{L}$, in order to choose $\mathbf{x}_{\|}$both as a local coordinate and as a variable parametrizing $\mathcal{L}$. Although this is not made explicit in our notations, different parametrizations $\mathbf{x}_{\|}$can be used for different contributions $\beta$.

Our results show that time evolution of mean values of operators in the semiclassical approximation cannot be obtained merely from the stationary phase approximation, at least when several ingredients are present. If the Wigner transform of the operator varies only at a classical scale, time evolution may stretch the manifold on which the semiclassical wave function is constructed in such a way that non-stationary points become relevant to the semiclassical dynamics. In that case, the stationary points (and their vicinity) only provide the classical contribution to the mean value. These stationary points correspond to cases where the chords joining a pair of points on the Lagrangian manifold become of length zero. The semiclassical contributions originate in the fact that small (at the quantum scale) but nonzero chords may arise, for instance when the dynamics is chaotic. These almost stationary points do not lie in the vicinity of a truly stationary point, so that the stationary phase approximation does not capture them.

Thus, when computing semiclassically the mean values of smooth operators for a semiclassical wavefunction one needs to bear in mind that, when short chords are involved, i.e. 
when the folding of the Lagrangian manifold $\mathcal{L}$ on which the wavefunction is built induces the presence of sheets that are extremely close from one another, $K$ integrals (over $\hat{\mathbf{x}}_{\perp}$ in our notation) can be performed within the stationary phase approximation; however the $K$ remaining ones (over $\mathbf{x}_{\|}$) do not involve fast oscillations or stationary phase points and must be performed by other means. The approach we suggest is thus to perform within the stationary phase approximation all the integrals that can be done in this way, and to perform the last remaining $K$ by other means, possibly numerically. One implication of this approach however is that it makes is necessary to identify on the initial manifold (at $t=0$ ) which points will end up close to each other at time $t$, which amount to a root search for the classical dynamics. This has to be contrasted with phase-space Initial (or Final) Value Representation (IVR or FVR) approaches which are specifically designed to avoid such root search.

These IVR/FVR semiclassical approaches are extremely popular in the chemical and molecular physics community, in particular in the version introduced by Herman and Kluck [31] which involve some degree of smoothing, but also in the original form introduced by Miller [32] for the van Vleck propagator or in the more modern and elegant form for the Wigner function introduced by Ozorio de Almeida and coworkers [14]. In these approaches, the classical trajectories implied in the semiclassical calculation under consideration are entirely specified by their initial conditions, thus avoiding the need of a root search. As a bonus one moreover usually avoids the divergences associated with caustics or turning points. The section 6 of [14] gives for instance an application to these IVR/FVR approaches to the calculations of mean values of operators that we have analyzed in this paper.

If the initial state $\left|\Psi_{0}\right\rangle$ that one is propagating does not correspond to a semiclassical wavefunction (in the form we define them in section II), there is no ambiguity that the IVR/FVR form of the semiclassical propagation of the mean value is superior to any approach that would imply a root search. If on the other hand $\left|\Psi_{0}\right\rangle$ does take the form of a semiclassical state, then IVR/FVR expressions for the operator mean value are of course equivalent (within stationary phase approximation) to inserting the semiclassical expression (19) into (4) 33. As expected, both describe the same physics, and deciding on one or the other is mainly a matter of effectiveness; the term of the choice being to do a root search or to avoid this root search at the cost of performing numerically extra integrals.

There is obviously a set of situations where avoiding the root search is enough of a 
simplification to justify the extra numerical integrals implied by the IVR/FVR approaches. However, if we go back to the calculations of transverse integral in section IV B 2 in the simple one-dimensional case, we see that this integral is dominated by a region of size $O\left(\sqrt{\hbar w^{\prime}}\right)$, with $w^{\prime}$ the variation of the distance between the two sheets of the manifold. As expected for semiclassical integrals evaluated at the stationary phase approximation, this size scales as $\sqrt{\hbar}$, and, as soon as one enters a bit deeply in the semiclassical regime, any attempt to compute numerically the integral will require a very fine grid to avoid the output being dominated by numerical noise. This state of things is quite general in semiclassical integrals which in the semiclassical limit are usually dominated by very small portion of configuration (or phase) space. Here, this is actually made worse by the factor $w^{\prime}$, which tends to zero when the two sheets of the manifold become parallel, which will necessarily happen when they become very close to each other. Even in the simple one-dimensional case, computing numerically the integral 30 in the deep semiclassical regime and for rather long evolution time of a chaotic system such that the folding of the manifold creates extremely close, almost parallel, pairs of sheets, will require to extract numerically the relevant information from a tiny area of phase space which, if the different role of the parallel and perpendicular directions are not recognized, will require to propagate classical trajectories on a grid on the sub-quantum scale in both directions. On the other hand our approach would in that case only require a one-dimensional numerical integration (along the manifold) with a grid on the classical scale.

We would thus argue that, if IVR/FVR are clearly the best options for semiclassical evolution of the mean value of an operator taken on a non-semiclassical wavefunction, and could be a viable route for short time / not too deeply semiclassic problems, the root search implied by our approach would still remain a considerably simpler and more accurate option in the deep quantum long-time chaotic regime.

As a final remark, in this paper we concentrated on the simplest form of folding of the classical manifold where, under chaotic time evolution, the Lagrangian manifold stretches out with a curvature on the classical scale. However much more involved classical structures can appear, such as the whorls and tendrils discussed in [30]. For us, it is an open question to decide whether the approach we propose can be adapted to these more complicated geometries, whether they could still be addressed semiclassically but would require numerical integration of the semiclassical expressions (bringing us back then to IVR/FVR), or whether 
they merely lead to diffractive effects that cannot be handled semiclassically.

\section{ACKNOWLEDGMENTS}

We are deeply thankful to Alfredo Ozorio de Almeida for the role he played in motivating this work, as well as in the many exchanges we had at later stages of the process.

This work was supported by the ANR COCOA project, grant ANR-17-CE30-0024-02 of the Agence Nationale de la Recherche.

[1] J. H. Van Vleck, Proceedings of the National Academy of Sciences of the United States of America 14, 178 (1928).

[2] P. A. M. Dirac, The principles of quantum mechanics, 27 (Oxford university press, 1981).

[3] M. C. Gutzwiller, Journal of Mathematical Physics 12, 343 (1971).

[4] M. C. Gutzwiller, Lecture Notes for the Summer School Les Houches (1989).

[5] V. Maslov and M. Fedoriuk, Semiclassical Approximation in Quantum Mechanics (Reidel, Dordrecht, 1981).

[6] L. Schulman, Techniques and Applications of Path Integration (John Wiley \& Sons, New York, 1981).

[7] R. A. Jalabert and H. M. Pastawski, Phys. Rev. Lett. 86, 2490âĂŞ2493 (20).

[8] N. R. Cerruti and S. Tomsovic, Phys. Rev. Lett. 88, 054103 (2002).

[9] S. Tomsovic, Phil. Trans. R. Soc. A 374, 20150161 (2016).

[10] J. Rammensee, J. D. Urbina, and K. Richter, Phys. Rev. Lett. 121, 124101 (2018).

[11] R. A. Jalabert, I. García-Mata, and D. A. Wisniacki, Phys. Rev. E 98, 062218 (2018)

[12] K. Richter and M. Sieber, Phys. Rev. Lett. 89, 206801 (2002).

[13] P. W. Brouwer and S. Rahav, Phys. Rev. B 74, 075322 (2006).

[14] A. M. Ozorio de Almeida, R. O. Vallejos, and E. Zambrano, Journal of Physics A: Mathematical and Theoretical 46, 135304 (2013).

[15] A. M. Ozorio de Almeida and O. Brodier, Journal of Physics A: Mathematical and Theoretical 49, $185302(2016)$.

[16] A. M. Ozorio De Almeida, Physics reports 295, 265 (1998). 
[17] P. d. M. Rios and A. Ozorio de Almeida, Journal of Physics A: Mathematical and General 35, 2609 (2002).

[18] H. Wang, X. Sun, and W. H. Miller, J. Chem. Phys. 108, 9726 (1998).

[19] A. Polkovnikov, Annals of Physics 325, 1790 (2010).

[20] E. J. Heller, The Journal of Chemical Physics 65, 1289 (1976).

[21] M. Hillery, R. F. O'Connell, M. O. Scully, and E. P. Wigner, Physics reports 106, 121 (1984),

[22] S. Tomsovic and E. J. Heller, Phys. Rev. Lett. 67, 664 (1991).

[23] D. Waltner, M. Gutiérrez, A. Goussev, and K. Richter, Phys. Rev. Lett. 101, 174101 (2008).

[24] G. M. Lando, R. O. Vallejos, G.-L. Ingold, and A. M. Ozorio de Almeida, Physical Review A 99, $042125(2019)$.

[25] M. V. Berry, Philosophical Transactions of the Royal Society of London. Series A, Mathematical and Physical Sciences 287, 237 (1977).

[26] S. Tomsovic, Physical Review E 98, 023301 (2018).

[27] A. Voros, Ann. Inst. Henri Poincaré 26, 343 (1977).

[28] A. Ozorio de Almeida and J. Hannay, Annals of Physics 138, 115 (1982).

[29] E. J. Heller, The Journal of Chemical Physics 67, 3339 (1977).

[30] M. V. Berry, Journal of Physics A: Mathematical and General 12, 625 (1979).

[31] M. F. Herman and E. Kluk, Chem. Phys. 91, 27 (1984).

[32] W. H. Miller, J. Chem. Phys. 53, 3578 (1970).

[33] K. M. Mittal, P. Haldar, O. Giraud, and D. Ullmo, unpublished (2020). 